\title{
Confirming the RNAi-mediated mechanism of action of siRNA-based cancer therapeutics in mice
}

\author{
Adam D. Judge, Marjorie Robbins, Iran Tavakoli, Jasna Levi, Lina Hu, \\ Anna Fronda, Ellen Ambegia, Kevin McClintock, and Ian MacLachlan
}

Tekmira Pharmaceuticals Corp., Burnaby, British Columbia, Canada.

\begin{abstract}
siRNAs that specifically silence the expression of cancer-related genes offer a therapeutic approach in oncology. However, it remains critical to determine the true mechanism of their therapeutic effects. Here, we describe the preclinical development of chemically modified siRNA targeting the essential cell-cycle proteins polo-like kinase 1 (PLK1) and kinesin spindle protein (KSP) in mice. siRNA formulated in stable nucleic acid lipid particles (SNALP) displayed potent antitumor efficacy in both hepatic and subcutaneous tumor models. This was correlated with target gene silencing following a single intravenous administration that was sufficient to cause extensive mitotic disruption and tumor cell apoptosis. Our siRNA formulations induced no measurable immune response, minimizing the potential for nonspecific effects. Additionally, RNAi-specific mRNA cleavage products were found in tumor cells, and their presence correlated with the duration of target mRNA silencing. Histological biomarkers confirmed that RNAi-mediated gene silencing effectively inhibited the target's biological activity. This report supports an RNAi-mediated mechanism of action for siRNA antitumor effects, suggesting a new methodology for targeting other key genes in cancer development with siRNA-based therapeutics.
\end{abstract}

\section{Introduction}

siRNAs are target-specific double-stranded RNA molecules designed to suppress gene expression through the endogenous cellular process of RNAi (1). Since the characterization of this fundamental gene-silencing mechanism, tremendous progress has been made in developing siRNA as a potentially novel class of therapeutic agent for a broad spectrum of diseases including cancer, viral infection, and metabolic disorders.

Many siRNA targets in oncology have been described in the literature, although direct evidence that their therapeutic effects in tumor models are mediated by RNAi is notably lacking. The interpretation of antitumor activity attributable to siRNAs is problematic due to the potential for off-target effects of the nucleic acids, including their propensity to activate immune responses through TLR-dependent (2-4) and TLR-independent mechanisms $(5,6)$. These types of response are known to elicit antitumor effects, primarily through the actions of IFNs and inflammatory cytokines that exert antiangiogenic, proapoptotic, and adjuvant effects that enhance cellular immunity $(7,8)$. Many of these mechanisms remain active in the immunodeficient mouse strains typically used as hosts for human tumor xenografts, including SCID/beige mice, which lack functional lymphocyte and NK cell populations $(9,10)$. Induction of the innate immune response by nucleic acids can also have significant toxicologic consequences (reviewed in

Conflict of interest: All authors are employees of Tekmira Pharmaceuticals Corp. Nonstandard abbreviations used: AS, antisense; bDNA, branched DNA; hGAPDH, human GAPDH; hPLK1, human PLK1; IFIT1, IFN-induced protein with tetratricopeptide repeats 1 ; KSP, kinesin spindle protein; 2'OMe, 2'-O-methyl; 2'OMe-G, 2'OMe-guanosine; 2'OMe-U, 2'OMe-uridine; PEG, poly(ethylene)glycol; PEG-cDMA, 3-N-(-methoxy poly(ethylene glycol)2000)carbamoyl-1,2-dimyristyloxy-propylamine; PEG-cDSA, 3-N-(-methoxy poly(ethylene glycol)2000)carbamoyl-1,2-distearyloxypropylamine; PLK1, polo-like kinase 1; RACE, rapid amplification of cDNA ends; RISC, RNA-induced silencing complex; RLM, RNA ligase mediated; RLU, relative light unit[s]; SNALP, stable nucleic acid lipid particle(s).

Citation for this article: J. Clin. Invest. 119:661-673 (2009). doi:10.1172/JCI37515. ref. 11). Clinical experience with certain recombinant cytokines and TLR agonists $(12,13)$ including liposomal plasmid DNA (I. MacLachlan, unpublished observations) has shown that human subjects can be exquisitely sensitive to the toxic effects of these agents when compared with preclinical models. Therefore additional caution is required if considering an immune stimulatory siRNA for clinical development $(14,15)$.

The incorporation of modified nucleotide chemistries into siRNA has been widely utilized to improve their pharmacologic and nuclease-resistant properties (16). We first reported that extensive chemical modification to siRNA molecules could provide the additional benefit of preventing their recognition by the mammalian immune system (17). This has led to the rational design of 2'-O-methyl-modified (2'OMe-modified) siRNA constructs that have inherently low immunostimulatory potential in vivo (18).

To establish proof that systemically administered siRNAs can elicit RNAi-mediated anticancer efficacy in the absence of measurable immune activation, we selected the essential cell-cycle proteins kinesin spindle protein (KSP, also referred to as Eg5) (19) and polo-like kinase 1 (PLK1) (20) as validated cancer targets with well-characterized mechanisms of direct tumor cell killing. KSP is a mitotic spindle motor protein that drives chromosome segregation during mitosis. Inhibition of KSP blocks the formation of bipolar mitotic spindles, causing cell-cycle arrest, activation of the mitotic checkpoint, and induction of apoptosis (21). In mammalian cells, PLK1 acts to phosphorylate a number of cell-cycle proteins including $\mathrm{Cdc} 25 \mathrm{C}$, cyclin B, cohesin subunit SCC-1, subunits of the anaphase promoting complex, mammalian kinesin-like protein 1, and other kinesin related proteins. This diverse array of substrates reflects the multiple roles of PLK1 in mitosis and cytokinesis (22). Overexpression of PLK1, observed in many human tumor types, is a negative prognosticator of patient outcome (reviewed in ref. 20), while inhibition of PLK1 activity rapidly induces mitotic arrest and tumor cell apoptosis $(23,24)$. Depletion 
of PLK1 may also sensitize cancer cells to the proapoptotic activity of small-molecule drugs (25), likely due to the role of PLK1 in the DNA damage and spindle assembly checkpoints.

One of the primary barriers to realizing the potential of siRNA therapeutics is the requirement for drug-delivery vehicles to facilitate disease site targeting, cellular uptake, and cytoplasmic delivery of the siRNA (26-28). Common approaches to delivery include complexing the siRNA with polycations such as polyethyleneimine $(29,30)$ and cyclodextrin polymers (31) as well as incorporation into cationic lipid-based carriers $(17,18,26,32)$. We have previously described the development of stable nucleic acid lipid particles (SNALP) as an effective systemic delivery vehicle for targeting siRNAs to the murine and nonhuman primate liver and have demonstrated therapeutic effects in silencing endogenous hepatocyte $(18,26)$ and viral gene transcripts (17). The accumulation of SNALP within tissues of clinical interest takes advantage of passive disease-site targeting $(33,34)$, whereby charge-neutral carriers of suitable size (around 100-nm diameter or smaller) can pass through the fenestrated epithelium of tumors, sites of inflammation, and the healthy liver. This avoids the requirement for active targeting moieties such as peptides, antibodies, and receptor ligands that may otherwise be candidates for incorporation into siRNA-delivery vehicles to enhance target-cell selectivity $(31,35,36)$.

In this report, we describe the preclinical development of SNALPformulated siRNAs as cancer therapeutics. Results demonstrate that rationally designed siRNAs targeting PLK1 or KSP, when delivered with an effective systemic delivery vehicle, are able to affect therapeutic gene silencing in solid tumors. The specificity and mechanism of action is confirmed using a combination of methodologies that demonstrate RNAi-mediated silencing of target mRNA causing mitotic disruption in tumor cells typical of target inhibition. This can be achieved in the complete absence of immune stimulation through the use of appropriately designed, chemically modified siRNAs.

\section{Results}

In vitro characterization of PLK1 siRNA activity. PLK1 represents a validated gene target in oncology whose inhibition is known to cause mitotic arrest and apoptosis in proliferating tumor-cell cultures (20). We designed and screened a panel of PLK1 siRNA for antiproliferative activity in the human HT29 colon cancer cell line (Supplemental Figure 1; supplemental material available online with this article; doi:10.1172/JCI37515DS1). This screen identified PLK1424 as the most potent human siRNA and PLK773 as the most potent mouse, rat, and human cross-reactive siRNA based on PLK1 sequence homology. These lead siRNAs were formulated into a SNALP composition that has been shown to effectively target siRNA to the livers of rodents and nonhuman primates (26). Treatment of HT29 cells with formulated PLK1424 and PLK773 siRNAs caused a dose-dependent decrease in cell viability that correlated with the degree of PLK1 mRNA silencing (Figure 1, A-C). PLK1424 siRNA displayed potent activity in a range of human cancer cell lines, including LS174T colon carcinoma and HepG2 and $\mathrm{He} 3 \mathrm{~B}$ hepatocellular carcinoma cell lines (Figure 1D), that was associated with the dose-dependent induction of apoptosis 48 hours after siRNA transfection (Figure 1E).

Design of PLK1 and KSP siRNA for in vivo applications. Prior to the in vivo assessment of synthetic siRNA, it is essential to anticipate the potential effects of immune stimulation on the biological system under consideration and take steps to mitigate this risk (11). We have previously reported that the selective introduction of 2 'OMe-guanosine (2'OMe-G) or $2^{\prime} \mathrm{OMe}$-uridine $\left(2^{\prime} \mathrm{OMe}-\mathrm{U}\right)$ residues into siRNA abrogates its capacity to activate an immune response $(18,37)$. This original strategy proposed restricting 2 'OMe modifications to the siRNA sense strand in order to minimize the potential of negatively impacting RNAi activity (18). While this approach remains broadly applicable for synthetic siRNA (37), we have found through extensions to our original studies that certain siRNA sequences incorporating a 2'OMe-modified sense strand, for example, the U(S)-ApoB1 duplex (18), may retain low-grade immunostimulatory activity. This was only evidenced by the upregulation of IFN-induced protein with tetratricopeptide repeats 1 (IFIT1) mRNA in the liver and spleen following i.v. administration of SNALP-formulated U(S)-ApoB1 siRNA in mice, despite there being no measurable serum cytokine response (Supplemental Figure 2). This residual IFIT1 induction, however, could be fully abrogated by the selective introduction of $2^{\prime} \mathrm{OMe}$ nucleotides to the antisense (AS) strand of the duplex (Supplemental Figure 2). These findings provided the rationale for our design and testing of 2'OMe siRNA against oncology targets.

A similar approach to siRNA design was applied to PLK1424 and PLK773 to generate duplexes that possessed no measurable immune stimulatory effects yet retained full RNAi activity. We regarded this step as a prerequisite to conducting in vivo studies in order to conclude the specificity of antitumor effects that may be observed. 2'OMe-U or 2'OMe-G nucleotides were substituted into the native sense and AS oligonucleotides to form a panel of modified PLK1424 and PLK773 duplexes (Table 1) that were then screened for the preservation of RNAi activity. 2'OMe-PLK1424 duplexes containing the modified AS strand A or B displayed antiproliferative activity similar to that of the native PLK1424 sequence when paired with either of the modified sense strands, 1 or 2 . In contrast, duplexes containing AS strand C lost significant activity, suggesting that this 2 'OMemodification pattern was poorly tolerated by the RNAi machinery (Figure 2A). The panel of 2'OMe-PLK773 duplexes displayed modest differences in activity compared with the native PLK773 sequence (Figure 2B). We selected PLK1424-2/A and PLK773-1/B siRNA duplexes (comprising the designated 2'OMe-modified sense/AS strands) for evaluation in an in vitro immune-stimulation model. As expected, native PLK1424 and PLK773 siRNAs and their constituent single-stranded RNAs (ssRNAs) stimulated murine Flt3ligand-derived dendritic cells to produce high levels of both IFN- $\alpha$ and IL-6 (Figure 2C), whereas this immune reactivity was completely abrogated in the PLK1424-2/A and PLK773-1/B duplexes.

To demonstrate the utility of this approach to siRNA design, we applied the same methodology to a published siRNA targeting KSP (38). The selected KSP siRNA (termed KSP2263 from its original description) has full sequence homology to mouse and human KSP mRNA and showed potent antiproliferative effects in both human and mouse cancer cell lines. As an example, treatment of mouse Neuro2a cells with SNALP-formulated KSP2263 induced dosedependent reductions in KSP mRNA 24 hours after transfection, correlating with a subsequent loss of cell viability at 72 hours (Figure 2D). A small panel of modified KSP2263 duplexes containing $2^{\prime} \mathrm{OMe}-\mathrm{U}$ or $2^{\prime} \mathrm{OMe}-\mathrm{G}$ nucleotides (Table 1 ) was then screened in this assay. In this case, each combination of the 2 modified sense and AS strands generated a duplex with potency equivalent to that of the native KSP2263 sequence, confirming preservation of RNAi activity (Figure 2E). We selected the 2'OMe-modified variant KSP2263-U/U for further characterization.

Confirmation of the RNAi mechanism by $5^{\prime} R A C E-P C R$. The detection of specific RNA cleavage products generated by RNA-induced silencing complex-mediated (RISC-mediated) hydrolysis of target 
A

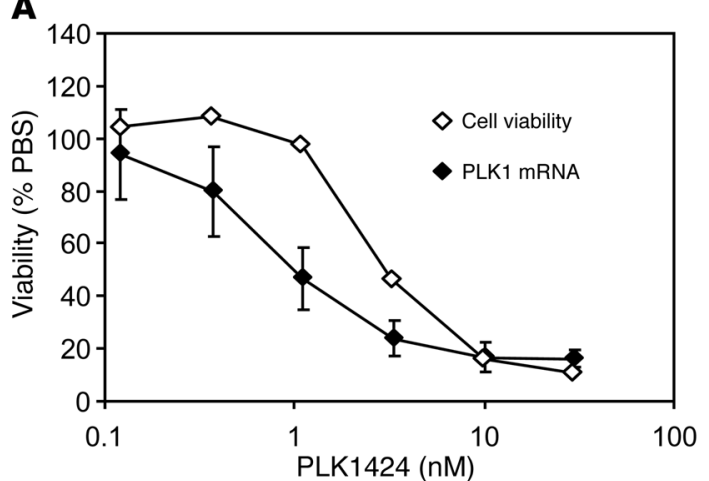

B

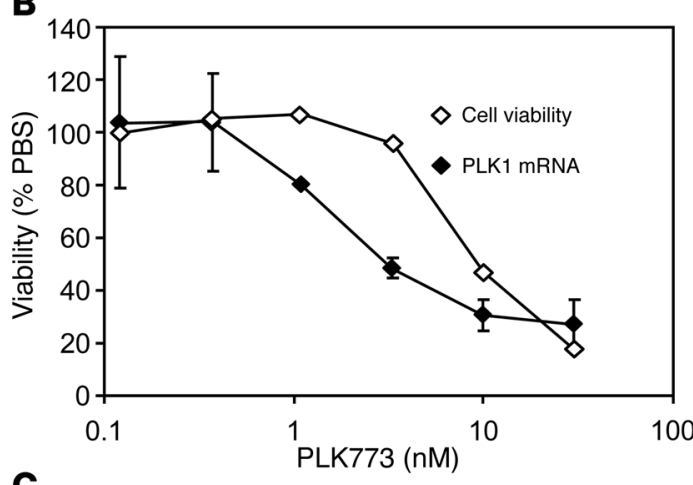

C

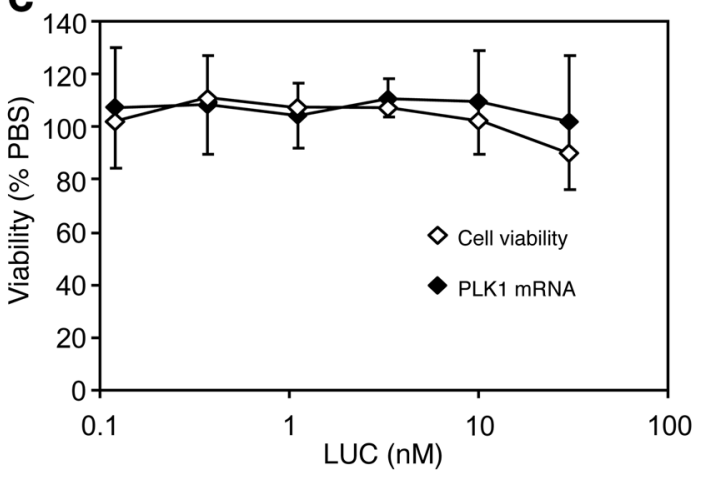

D
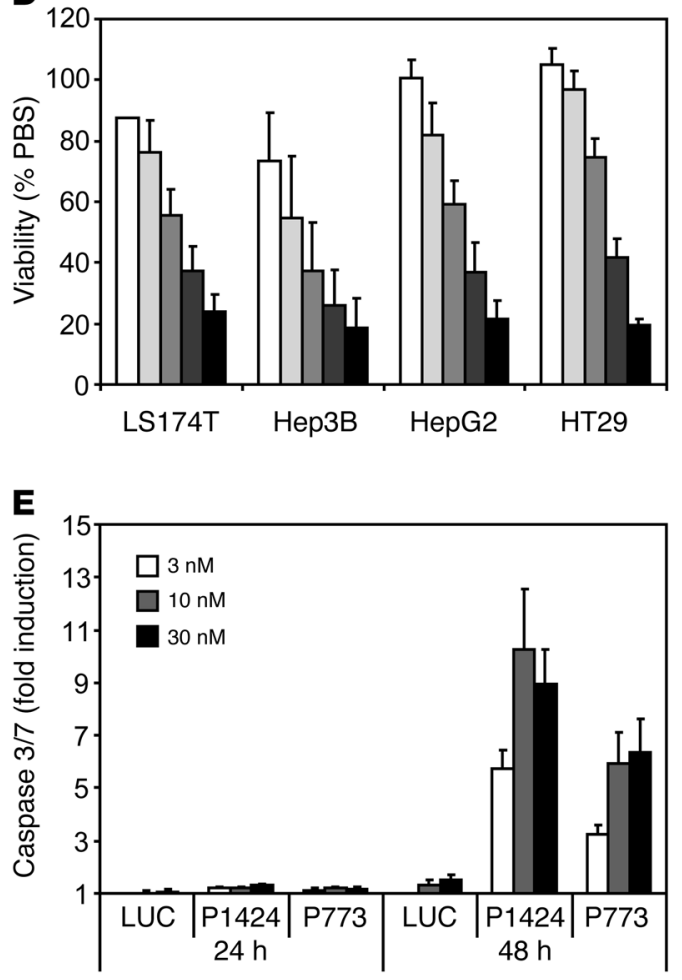

Figure 1

Activity of PLK1 siRNAs in vitro. Correlation between mRNA silencing and HT29 cell viability for (A) PLK1424 (P1424), (B) PLK773 (P773), and (C) LUC siRNA treatments. PLK1 mRNA was determined by bDNA analysis at 24 hours. Duplicate plates were assessed for cell viability at 72 hours. (D) PLK1424 siRNA causes dose-dependent reductions in viability of LS174T, HT29, Hep3B, and HepG2 cell cultures. Cells were treated for 72 hours with PLK1424 SNALP at increasing concentrations of $0.3,0.6,1.25,2.5$, and $5 \mathrm{nM}$ siRNA (white bars to black bars, respectively). Values in A-D are expressed as percentage of viability or PLK1 mRNA relative to PBS control and reflect mean of 3 separate experiments ( \pm SD) conducted in triplicate cultures. (E) Decreased cell viability is associated with the induction of apoptosis. Caspase-3/7 activity in LS174T cells was assessed 24 hours and 48 hours after treatment with SNALP-encapsulated PLK773, PLK1424, and LUC control siRNAs. Data represent fold induction over PBS in triplicate cultures (mean \pm SD triplicate cultures).

mRNA is the definitive marker confirming RNAi as the mechanism of gene silencing $(39,40)$. Activated RISC cleaves target mRNA precisely between the nucleotides complementary to positions 10 and 11 of the siRNA AS strand, generating an mRNA cleavage product that is unique to the siRNA sequence. This can be detected in cells using an appropriately designed $5^{\prime}$ rapid amplification of cDNA ends-PCR method (RACE-PCR). We developed RACE-PCR assays to detect the PLK1424-specific cleavage product of human PLK1 (hPLK1) mRNA and the KSP2263-specific cleavage product of mouse KSP mRNA. Treatment of HT29 cells with PLK1424-2/A generated the predicted 476-bp 5' RACE-PCR product, and oligonucleotide sequencing across the $5^{\prime}$ ligation site confirmed its identity as the hPLK1 mRNA product cleaved at $5^{\prime}$ position 1433 (relative to ATG start site). Similarly, a predicted 102-bp RACEPCR product was amplified from Neuro2a cells treated with KSP2263-U/U siRNA that corresponded to mouse KSP mRNA cleaved at position 2129. (Supplemental Figure 3).

Characterization of the immune response to $2^{\prime} \mathrm{OMe}$ PLK1 and KSP siRNA in vivo. To confirm the abrogation of immune stimulation by $2^{\prime} \mathrm{OMe}$ siRNA in vivo, $\mathrm{BALB} / \mathrm{c}$ mice were treated i.v. with 


\begin{tabular}{|c|c|c|}
\hline Name & Strand & Sequence (5'-3' 21-mer) \\
\hline PLK1424 & S & AGAUCACCCUCCUUAAAUAUU \\
\hline PLK1424 & AS & UAUUUAAGGAGGGUGAUCUUU \\
\hline PLK1424-1 & S & AGAUCACCCUUCCUUAAAUAUU \\
\hline PLK1424-2 & $S$ & 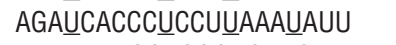 \\
\hline PLK1424-A & AS & UAUUUAAGGAGGGUGAŪCUUU \\
\hline PLK1424-B & AS & UAUUUAAGGGAGGGUGAŪCUUU \\
\hline PLK1424-C & AS & UAUUUAAGGAGGGGUGAUUCUUU \\
\hline PLK773 & S & AGACCUACCUCCGGAUCAAUU \\
\hline PLK773 & AS & UUGAUCCGGAGGUAGGUCUUU \\
\hline PLK773-1 & $S$ & AGACCUACCUCCGGAUCAAUU \\
\hline PLK773-2 & S & AGACCUACCUECCGGAUCAAUU \\
\hline PLK773-A & AS & 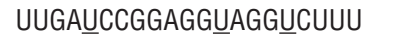 \\
\hline PLK773-B & AS & UUGAŪCCGGAGGŪAGGŪCUUU \\
\hline PLK773-C & AS & UUGAUCCGGGAGGUAGGUCUUU \\
\hline KSP2263 & $S$ & CUGAAGACCUGAAGACAAUdTdT \\
\hline KSP2263 & AS & AUUGUCUUCAGGUCUUCAGdTdT \\
\hline KSP2263-U & S & 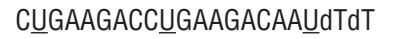 \\
\hline KSP2263-G & S & CUGAAGACCUGAAGACAAUdTdT \\
\hline KSP2263-U & AS & 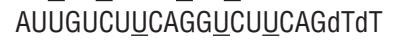 \\
\hline KSP2263-G & AS & AUUGUCUUCAGGŪCUUCAGdTdT \\
\hline LUC & $S$ & GAUUAUGUCCGGUUAUGUAUU \\
\hline LUC & AS & UACAUAACCGGACAUAAUCUU \\
\hline LUC-U & S & 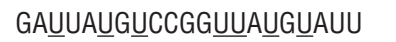 \\
\hline LUC-U & AS & UACAUAACCGGACAUAAUCUU \\
\hline
\end{tabular}

Underlines indicate 2'OMe nucleotides. known to protect unmodified siRNA from nuclease degradation for more than 24 hours in serum (18). However, the 2'OMe modification pattern can take into account the avoidance of (a) position 9 in the sense strand based on the observation that efficient activation of RISC involves initial cleavage of the siRNA sense strand between positions 9 and 10 and this can be inhibited by the introduction of nuclease-resistant chemistries at this linkage (41, 42); and (b) the 5' AS terminus where modified chemistries may interfere with effective RNA loading into RISC $(43,44)$.

Therapeutic inhibition of tumor growth by systemic siRNA administration. We established orthotopic liver tumor models to examine the pharmacodynamics and therapeutic efficacy of SNALP-formulated PLK1424-2/A and KSP2263-U/U siRNA. These were a Hep3B xenograft in SCID/beige mice as a representative model of human hepatocellular carcinoma and a syngeneic Neuro2a tumor model in immune-competent A/J mice. Tumor cells were injected directly into the left lateral liver lobe to establish primary intrahepatic tumors (45). This procedure resulted in histologically distinct, localized tumor nodules in more than $90 \%$ of mice in both models.

To evaluate the therapeutic efficacy of SNALP-formulated PLK1 siRNA, mice bearing established Hep3B liver tumors were treated with $2 \mathrm{mg} / \mathrm{kg}$ PLK1424-2/A or LUC-U/U siRNA by i.v. administration twice weekly for 3 weeks, until control groups displayed symptoms of extensive tumor burden. We have found progressive body weight loss to be a good indicator of hepatic tumor burden in the Hep3B-SCID/beige mouse model. Weight loss in LUC-U/Utreated mice was evident $12-16$ days after tumor implantation and proceeded throughout the remainder of the study (Figure 4A). In contrast, PLK1424-2/A SNALP-treated mice typically maintained body weight over the duration of treatment, indicating that the siRNA formulation was well tolerated and suggesting therapeutic benefit. A humane end point was defined according to daily clinical scores that were an aggregate of weight loss, body condition, and abdominal distension. In this aggressive orthotopic model, the time until first euthanization in the LUC-U/U group was 28 days after tumor seeding, with a median survival time of 32 days. By comparison, the times to first euthanization and median survival in the PLK1424-2/A SNALP-treated mice were significantly extended, to 44 days and 51 days, respectively $(P<0.05$; Figure $4 \mathrm{~B})$. Similar extensions of survival times were observed in repeat studies utilizing athymic nu/nu mice as hosts (Supplemental Figure 4).

The extent of Hep3B liver tumor burden was then assessed at the completion of dosing with PLK1424-2/A on day 22 after tumor implantation (1 day after the fifth siRNA dose). At autopsy, only 2 of 6 PLK1424-2/A-treated mice had visible tumors localized around the site of cell implantation into the liver lobe compared with extensive macroscopic tumor burden in corresponding control animals (Supplemental Figure 5). Species-specific probe sets to human GAPDH (hGAPDH) mRNA detected low levels of this tumor-derived signal in 5 of 6 PLK1424-2/A-treated mice, ranging from 2- to 6-fold above the background signal from normal mouse liver (Figure 4C), indicating that tumor growth was significantly suppressed but not completely eradicated by this treatment regime.

To examine more closely the tolerability of systemic siRNA administration, we conducted multidose toxicity studies using the mouse surrogate PLK773-1/B. Repeat administration of SNALP-formulated PLK773-1/B at $2 \mathrm{mg} / \mathrm{kg}$, twice weekly (mirroring the therapeutic dosing regimen) caused no significant changes in serum liver enzyme levels, total wbc counts, lymphocyte and neutrophil counts, platelet numbers, or rbc parameters assessed after 15 and 29 days of continu- 
A

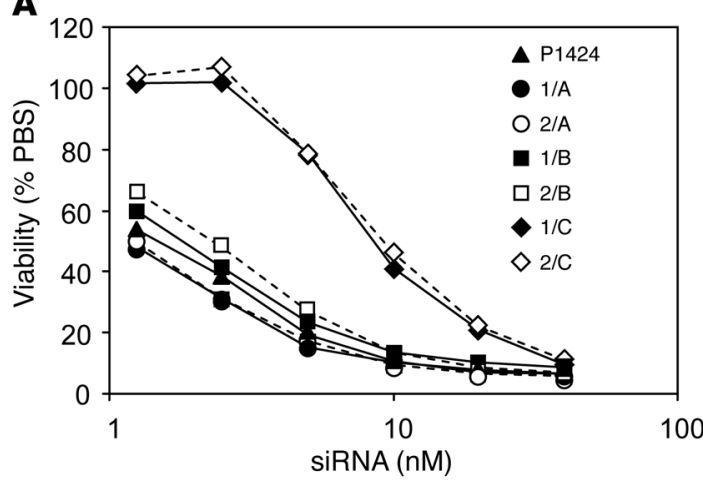

B
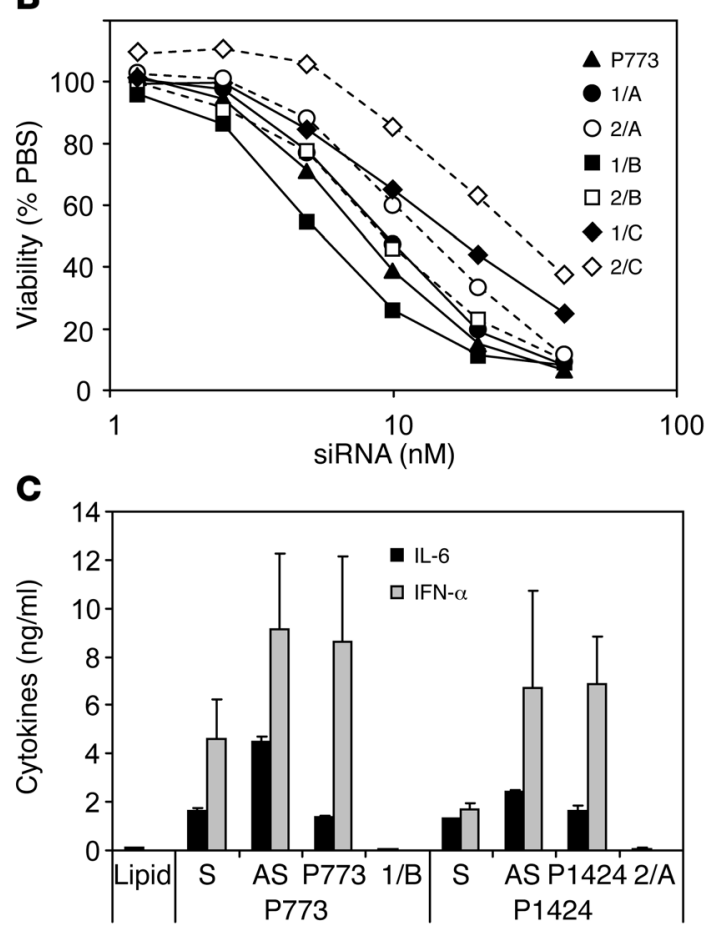

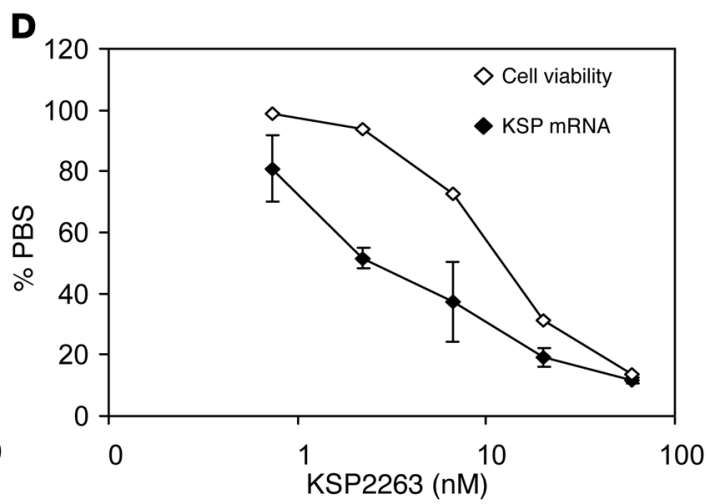

E

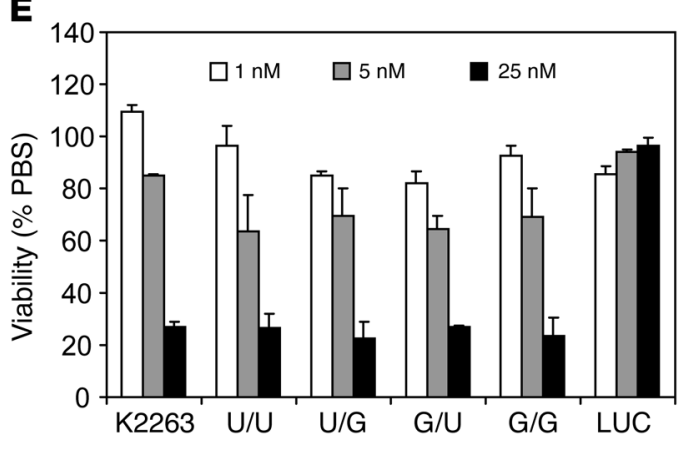

\section{Figure 2}

In vitro activity of unmodified versus $2^{\prime}$ OMe-modified PLK1 and KSP siRNA. Activity of the 2'OMe-modified panels of (A) PLK1424 and (B) PLK773 siRNA. Unmodified PLK1424 or PLK773 siRNA was compared in the Hep3B cell viability assay with the 2'OMe-modified duplexes 1/A, $2 / A, 1 / B, 2 / B, 1 / C$, and 2/C that comprise the respective 2'OMe sense/AS oligonucleotides (see Table 1). Data show mean viability of triplicate cultures relative to PBS-treated cells and represent 2 independent experiments using SNALP-formulated siRNAs. (C) Cytokine induction by unmodified and 2'OMe PLK1 siRNA in vitro. Murine Flt3L DCs were treated with $5 \mu \mathrm{g} / \mathrm{ml}(350 \mathrm{nM})$ unmodified PLK773 and PLK1424 siRNA duplexes and their constituent sense (S) or AS oligonucleotides or the 2'OMe siRNA duplexes PLK773-1/B (1/B) and PLK1424-2/A (2/A) formulated in SNALP. IFN- $\alpha$ and IL-6 were assayed in culture supernatants at 24 hours. Values represent mean + SD of 3 separate experiments conducted in triplicate cultures. (D and E) Activity of SNALP-formulated KSP2263 siRNA in murine Neuro2a cells. (D) Correlation between KSP mRNA silencing and cell viability relative to PBS control. KSP mRNA was determined by bDNA analysis at 24 hours. Duplicate plates were assessed for cell viability at 72 hours. (E) Activity screen comparing the unmodified KSP2263 siRNA to KSP2263-U/U (U/U), KSP2263-G/U $(\mathrm{G} / \mathrm{U})$, and KS2263-G/G (G/G) siRNA duplexes that comprise the respective sense/AS 2'OMe oligonucleotides (see Table 1). SNALP-formulated KSP2263 siRNA were tested in the Neuro2a cell viability assay. Data represent mean \pm SD triplicate cultures, relative to PBS treatment.

ous treatment (Supplemental Figure 6). These results indicate that the therapeutic dosing regime established in the orthotopic tumor model caused minimal hepatocellular toxicity and no significant bone marrow dysfunction of the type frequently observed with the systemic administration of small-molecule antimitotic drugs.

We next evaluated the therapeutic effect of SNALP-formulated KSP2263-U/U siRNAs in syngeneic Neuro2a liver tumors. Median survival time of mice receiving LUC-U/U SNALP $(4 \mathrm{mg} / \mathrm{kg}, \mathrm{Q} 3 \mathrm{~d} \times 5)$ was 20 days in this model compared with 28 days in the KSP2263-U/U treatment group (Figure 4D), demonstrating therapeutic efficacy with SNALP-formulated siRNAs for a second oncology target.

Confirmation of RNAi-mediated tumor gene silencing in vivo. Despite demonstrating that the 2 'OMe siRNA did not induce a measurable immune response in mice, it remained critical to show that RNAi was the primary mechanism underlying the potent therapeutic effects of these PLK1 and KSP siRNA formulations. A 
A

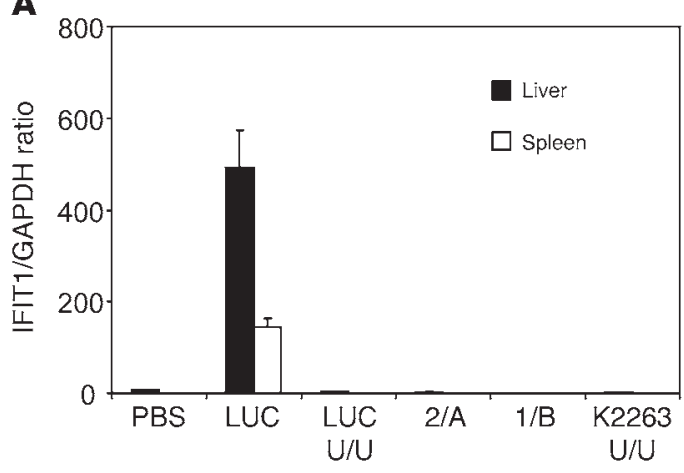

B

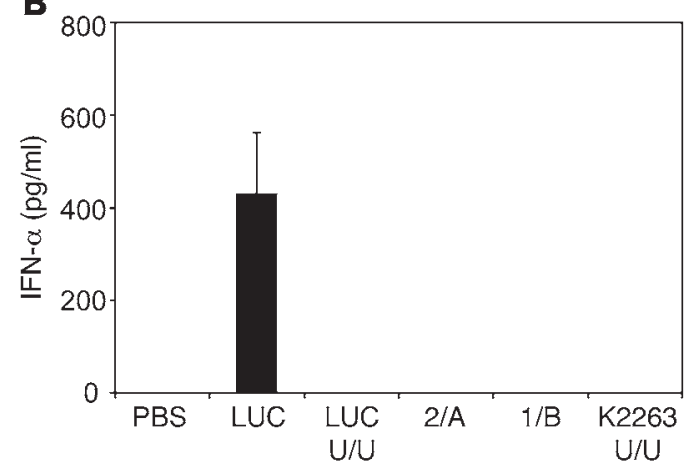

Figure 3

2'OMe-modified PLK1, KSP, and LUC siRNA induce no measurable IFN response in mice. SNALP-formulated LUC (unmodified) and 2'OMemodified LUC-U/U, PLK1424-2/A, PLK773-1/B, and KSP2263-U/U (K2263 U/U) siRNA were administered at 2 mg/kg i.v. to BALB/c mice. (A) IFIT1 relative to GAPDH mRNA in liver and spleen was assessed at 4 hours by bDNA analysis. (B). Serum IFN- $\alpha$ was assessed at 6 hours by ELISA. SNALP-formulated 2'OMe siRNAs induced no detectable increase in either IFN- $\alpha$ protein or IFIT1 mRNA relative to PBS vehicle. Values represent mean $+\operatorname{SD}(n=4)$.

single i.v. administration of SNALP-formulated PLK1424-2/A $(2 \mathrm{mg} / \mathrm{kg})$ caused a significant reduction in tumor-derived hPLK1 mRNA in hepatic hep3B tumors 24 hours after administration $(45 \% \pm 6.8 \%$ of hPLK1 mRNA levels in PBS-treated mice; Figure $5 \mathrm{~A}$ ). A similar reduction in mouse KSP mRNA expression was achieved using an equivalent dose of KSP2263-U/U in the hepatic Neuro2a tumor model (Figure 5B). In contrast to KSP and PLK1 expression in tumors, endogenous expression of both these genes in the surrounding nonproliferative liver was found to be very low, below the level of detection of the branched DNA (bDNA) assay employed in these studies (A. Judge, unpublished observations). Since the expression of cell-cycle genes such as PLK1 and KSP is typically downregulated as cells exit the cell cycle (22), any nonspecific, antiproliferative effects induced by siRNA or the delivery vehicle would cause a general decrease in their expression within tumors. We therefore confirmed RNAi as the mechanism responsible for mRNA silencing in vivo by the 5 ' RACE PCR method. A PCR product of the predicted size was readily amplified from hepatic Hep3B tumor samples taken 24 hours after administration of PLK1424-2/A SNALP (Figure 5C). Oligonucleotide sequencing of the 476-bp PCR product from 3 individual mice confirmed its identity as the predicted $5^{\prime}$ cut end of hPLK1 mRNA. This PCR product was not evident in tumors taken from LUC-U/U siRNA-treated mice or in liver samples from non-tumor bearing animals. RACE-PCR analysis also confirmed the specific induction of RNAi-mediated KSP mRNA cleavage within tumors of KSP2263-U/U-treated animals (Figure 5D).

5 'RACE-PCR to monitor the duration of RNAi in tumors. To determine the duration of active RNAi within the tumor, we treated a cohort of Hep3B tumor-bearing mice with PLK1424-2/A SNALP $(2 \mathrm{mg} / \mathrm{kg}$ by i.v. administration) and collected tumors 24 hours, 48 hours, 96 hours, 7 days, and 10 days after administration for analysis by $5^{\prime}$ RACE-PCR. Active PLK1 mRNA cleavage remained strong at 48 and 96 hours and was still evident 7 days after a single siRNA administration. A weak signal was detected in PLK1424-treated animals on day 10 (Figure 6A). The duration of RNAi determined by RACEPCR closely correlated with the level of hPLK1 mRNA silencing in these liver tumors (Figure 6B), providing further confirmation that RNAi was the primary mechanism for reductions in PLK1 mRNA.
Since the cleaved mRNA species are inherently unstable in the cell cytoplasm, it can be concluded that active RISC-mediated cleavage of the target mRNA persisted for 7-10 days after a single siRNA treatment. This suggests that active RNAi continued to occur either within a subset of tumor cells at subcytotoxic levels or within an initially nonproliferative population that subsequently entered cell cycle and reexpressed PLK1 mRNA.

RNAi-mediated antitumor activity assessed by histology. Many antimitotic drugs, including KSP (46) and PLK1 inhibitors (47, 48), induce distinct nuclear phenotypes that reflect their underlying mechanism of action. We therefore used conventional histology as a biomarker to assess whether the degree of RNAi-mediated gene silencing in vivo was sufficient to induce the desired antimitotic effect in tumor cells. Inhibition of KSP prevents bipolar spindle formation and centrosome segregation, leading to the formation of characteristic monoastral spindles. We first confirmed that the treatment of tumor cells with KSP2263-U/U siRNA induced the distinct monoastral nuclear phenotype in vitro (Supplemental Figure 7). Conventional histology on Neuro2a tumors from KSP2263-U/Utreated mice revealed significant numbers of tumor cells with aberrant mitotic figures typical of monoastral and apoptotic cells (46) 24 hours after SNALP administration (Figure 7, A and B). This dramatic pharmacodynamic response to KSP2263-U/U treatment was dose dependent, with maximal effects observed at $2 \mathrm{mg} / \mathrm{kg}$ siRNA, based on quantitative histology scores (Figure 7C). This analysis demonstrated that approximately $13 \%$ of total Neuro2a tumor cells displayed condensed chromatin structures at 24 hours after siRNA treatment compared with approximately $3 \%$ of cells displaying typical mitotic figures in control tumors.

Histological analysis of Hep3B liver tumors from PLK1424-2/A SNALP-treated mice also revealed abundant tumor cells with condensed chromatin structures and aberrant mitotic figures (Figure 8). These phenotypic changes were consistent with the dysregulated chromosomal segregation and apoptosis that is induced by PLK1 inhibition (47) and were in striking contrast to the typical mitotic figures evident in the tumor histology of control-treated animals.

These molecular and cellular pharmacodynamic studies confirmed that the degree of RNAi-mediated silencing achieved by a single i.v. administration of SNALP-formulated PLK or KSP siRNA 
A

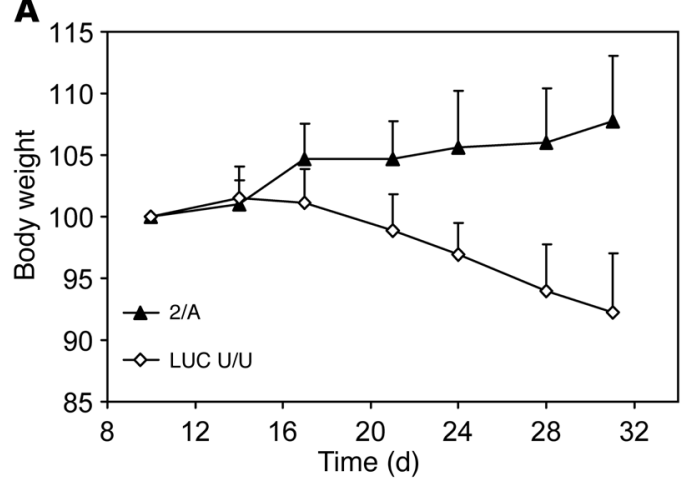

B

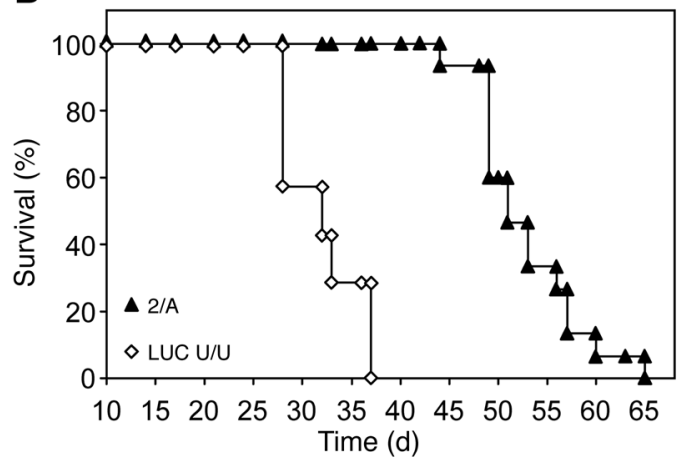

C

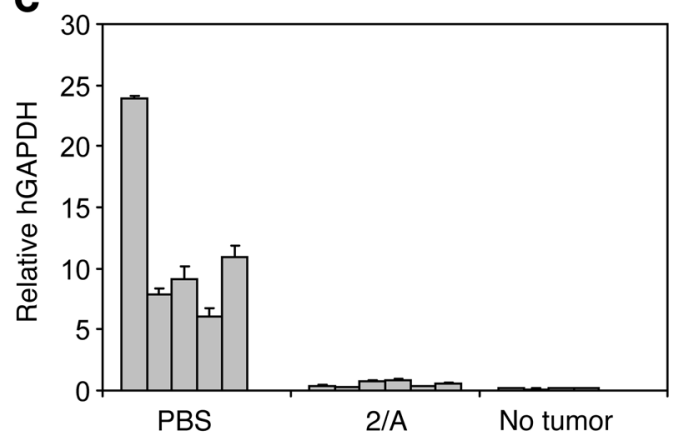

D

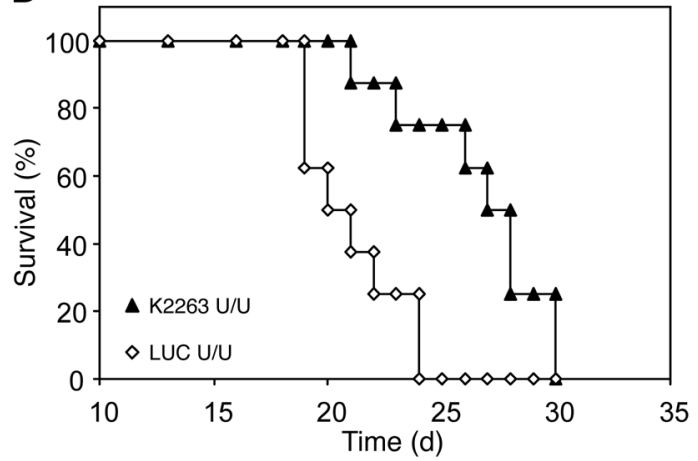

Figure 4

Therapeutic activity of PLK1 and KSP siRNA in hepatic tumors. PLK1424-2/A treatment confers significant survival advantages in SCID/beige mice bearing hepatic Hep3B tumors. Mice were administered SNALP-formulated PLK1424-2/A ( $n=15)$ or LUC-U/U $(n=8)$ at $6 \times 2$ mg/kg i.v. twice weekly (day 10 to day 28). (A) Body weights (mean + SD) over the dosing period expressed as percentage of initial weight on day 10. (B) Kaplan-Meier plot of days to euthanization due to tumor burden. PLK1424-2/A treatment provided significant survival advantage over control treatment. ( $P=0.03$, log-rank Cox-Mantel test). (C) Residual hepatic Hep3B tumor burden in mice 24 hours after final administration of PLK1424-2/A siRNA $(5 \times 2 \mathrm{mg} / \mathrm{kg}$ siRNA on days $8,11,14,18$, and 21$)$. Bars represent hGAPDH mRNA/mg liver of individual mice (mean \pm SD of triplicate analyses) determined by human-specific bDNA assay. No tumor, livers from non-tumor-seeded mice. See Supplemental Figure 6 for additional data. (D) KSP2263-U/U treatment confers survival advantages in A/J mice bearing hepatic Neuro2a tumors. Mice were administered SNALPformulated KSP2263-U/U or LUC-U/U $(n=8)$ at $5 \times 4 \mathrm{mg} / \mathrm{kg}$ i.v. (q3d $\times 5$ from day 8 to day 21 after tumor seeding). Kaplan-Meier plot of days to euthanization due to tumor burden. End points are based on clinical scores as a humane surrogate for survival. Mean SNALP particle sizes were 83 (0.09 polydispersity), and 90 (0.12 polydispersity) nm for PLK1424-2/A and LUC-U/U formulations, respectively.

was sufficient to cause mitotic dysfunction in a substantial proportion of tumor cells. Histological assessments of drug activity in both models demonstrated that "affected" cells were distributed throughout established tumors, indicating good penetration of the lipidic delivery vehicle. Taken together, this battery of tests provided conclusive evidence that the potent therapeutic effects of these SNALP-formulated siRNAs in the absence of a measurable immune response are the result of RNAi.

Therapeutic activity of SNALP-formulated siRNA in s.c. tumors. To expand the general utility of this technology in oncology, we determined whether the performance of this liver-targeting SNALP formulation (26) could be further improved for delivering siRNA to tumors outside of the liver. For vehicles containing poly(ethylene)glycol-conjugated lipids (PEG-lipids) such as SNALP, increased blood residency time and tumor accumulation can be achieved by incorporating PEG-lipids with longer alkyl chains that associate more strongly with the lipid particle and provide greater shielding in the blood compartment (49). Replacing the C14 PEG-lipid (3-N-(-methoxy poly(ethylene glycol)2000)carbamoyl-1,2-dimyrestyloxy-propylamine [PEGcDMA]) with the C18 analogue 3-N-(-methoxy poly(ethylene glycol)2000)carbamoyl-1,2-distearyloxy-propylamine (PEG-cDSA) (50) had the predicted effect of significantly increasing the blood circulation time of PLK1424-2/A SNALP in mice without altering its therapeutic efficacy in hepatic tumors (Supplemental Figure 8; median survival: PLK PEG-cDMA, 51 days; PLK PEG-cDSA, 53 days versus LUC; PEG-cDMA, 33 days; $P<0.05)$.

Despite a relatively short blood-circulation time and rapid distribution to the liver, repeat administration of PEG-cDMA SNALP containing PLK1424-2/A caused significant inhibition of s.c. Hep3B tumor growth compared with LUC-U/U siRNA treatment controls (Figure 9A). PLK1424-2/A formulated in an equivalent PEG-cDSA SNALP exhibited more potent antitumor effects, inducing regression of established tumors $(\sim 7 \mathrm{~mm}$ diameter) through the dosing period (Figure 9A). This difference in activity correlated with the degree of gene silencing induced by these PLK1424-2/A SNALP in s.c. tumors (Figure 9B). As in the hepatic tumor models, this was confirmed as being mediated by RNAi by both RACE-PCR and tumor histology (A. Judge, unpublished observations). Finally, we established the therapeutic dose response of the PEG-cDSA PLK1424-2/A formulation in the s.c. model. Dose-dependent inhibition of tumor growth was evident 
A

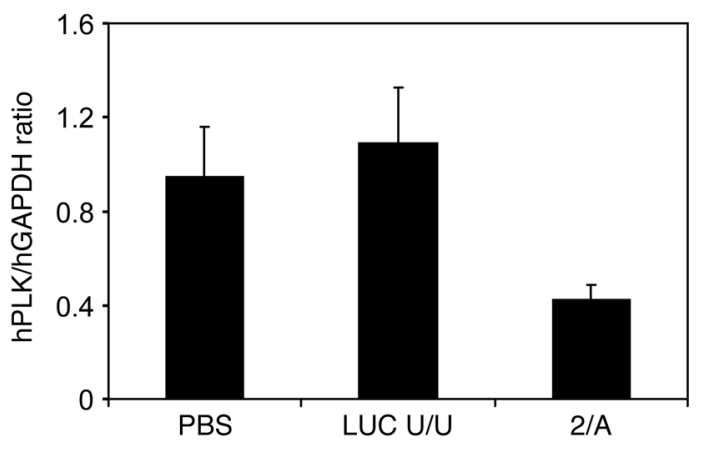

B

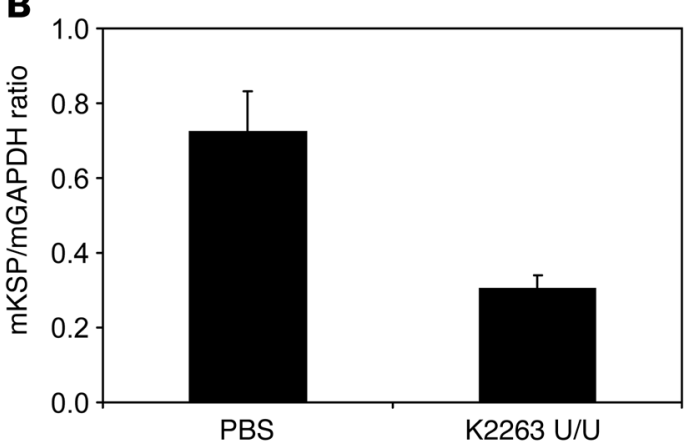

C

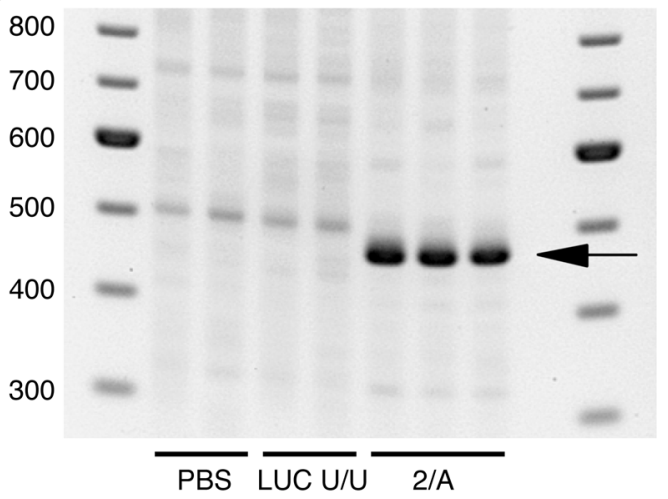

D

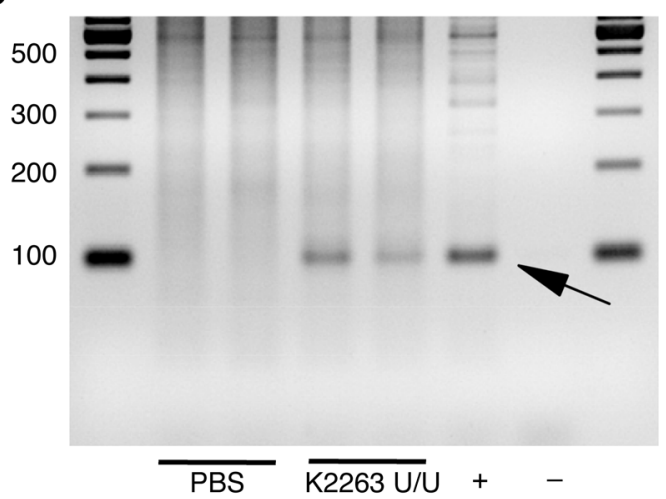

Figure 5

Target mRNA silencing in hepatic tumors by the RNAi mechanism. (A and B) Target mRNA silencing and (C and D) detection of RNAi-specific mRNA cleavage products in tumors following SNALP-formulated siRNA administration. SCID/beige mice with established intrahepatic Hep3B tumors were administered a single $2 \mathrm{mg} / \mathrm{kg}$ dose of SNALP-formulated PLK1424-2/A or LUC-U/U siRNA, and RNAi activity was assessed by (A) PLK1 mRNA in tumor lysates and (C) 5' RACE-PCR analysis. (A) Tumor (human) PLK1/GAPDH mRNA ratios 24 hours after siRNA administration (mean \pm SD of 4 animals). (C) RACE-PCR detects the specific $5^{\prime}$ cleavage product of PLK1 mRNA from tumors analyzed in A. Lanes represent PCR products derived from individual PBS $(n=2)$, LUC-U/U $(n=2)$, and PLK1424-2/A-treated mice $(n=3)$. (B) Mouse KSP mRNA and (D) 5' RACE-PCR analysis of resected hepatic Neuro2a tumors from mice treated with SNALP-formulated KSP2263-U/U siRNA. Data are presented as in A and C. Positive control from in vitro Neuro2a cell lysates treated with KSP2263-U/U siRNA indicated by plus sign; no template control indicated by minus sign. RACE-PCR detects the specific 5' cleavage product of mouse KSP mRNA from tumors. Identities of the predicted 476-bp PLK1 and 102-bp KSP PCR products (arrows) were confirmed by direct DNA sequencing. Mean SNALP particle sizes were 83 (0.09 polydispersity), 90 (0.12 polydispersity), and 88 (0.07 polydispersity) nm for PLK1424-2/A, LUC-U/U, and KSP2263-U/U formulations, respectively.

from 0.5 to $3.0 \mathrm{mg} / \mathrm{kg}$ PLK1424-2/A siRNA (Figure 9C). At the lowest dose level tested, this represented a total cumulative dose of $3 \mathrm{mg} / \mathrm{kg}$ siRNA over a 2 -week period.

\section{Discussion}

Delineating the mechanism of action for nucleic acid-based drugs has historically been confounded by underlying immune stimulation or other nonspecific effects induced by the nucleic acid $(51,52)$. This remains a valid concern for the burgeoning field of siRNA-based therapeutics (11). Assessment of target mRNA or protein downregulation is necessary but not sufficient to conclude that RNAi is the underlying mechanism, as these changes may also be symptomatic of the off-target effects induced by siRNA. In this report on the development of SNALP-formulated siRNA for oncology applications, we describe the methodology used to confirm both the specificity and mechanism of action underlying the potent siRNA-mediated antitumor efficacy in preclinical models. This involved a combination of approaches: first, the design of both active and control siRNA formulations with no apparent capacity to activate an immune response, therefore excluding as best as possible the potential for nonspecific efficacy; second, the selection of validated oncology targets (PLK1 and KSP) with direct antitumor effects and distinctive histological biomarkers of functional target inhibition; third, the use of RACE-PCR to confirm induction of the RNAi-specific mRNA cleavage product in tumor cells; and fourth, the correlation of this active RNAi signature with the duration of target mRNA silencing in tumors. We believe that this is the first report describing antitumor effects of siRNA to formally demonstrate RNAi as the primary mechanism of action. Furthermore, this approach to preclinical study design can be generalized to other targets in oncology and readily adopted by researchers in the RNAi field.

To evaluate the therapeutic potential of gene silencing in tumors without the confounding effects of immune stimulation, we designed 2'OMe-modified siRNA that completely abolished the immunostimulatory activity of unmodified (native) RNA duplexes when administered in a delivery vehicle. It is well established that the large majority of native siRNA duplexes have the inherent capac- 
A

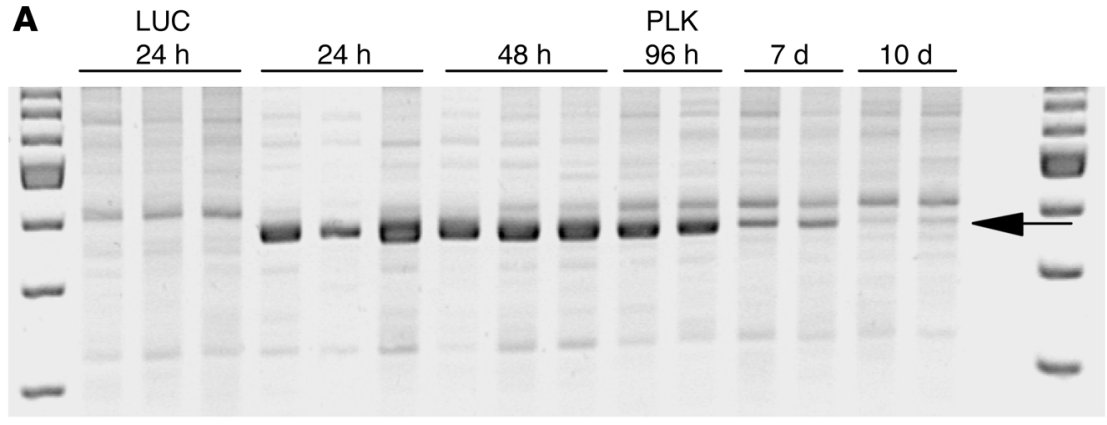

B

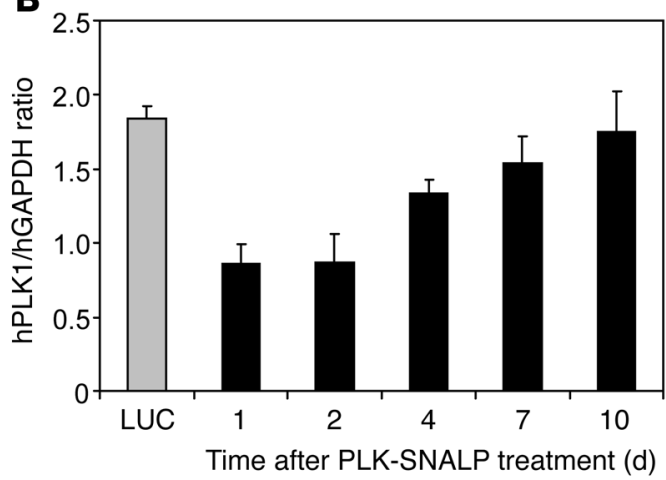

\section{Figure 6}

Duration of RNAi activity within hepatic tumors. (A) 5' RACE-PCR analysis of Hep3B liver tumors 24 hours, 48 hours, 96 hours, 7 days, and 10 days after a single i.v. administration of SNALP-formulated PLK1424-2/A siRNA (2 mg/kg). Specificity of the PLK1424-specific RACE-PCR product (arrow) was confirmed by sequencing at day 1 and day 7. (B) Corresponding levels of PLK1 mRNA in isolated tumor RNA analyzed in A. Duration of RNAi correlated with duration of mRNA silencing compared with that of LUC-U/U SNALP-treated mice. Data represent mean hPLK1/ hGAPDH mRNA ratio + SD ( $n=3$ at each time point). Mean SNALP particle sizes were 83 ( 0.09 polydispersity) and 90 (0.12 polydispersity) $\mathrm{nm}$ for PLK1424-2/A and LUC-U/U, respectively. ity to activate the innate immune response through the endosomal TLR7 and/or TLR8 pathway, particularly when cellular uptake is facilitated by delivery vehicles $(2,3,18,53)$. Naked (nonformulated) siRNA duplexes of $21 \mathrm{bp}$ or longer have also been reported to activate cell-surface TLR3 on endothelial cells, causing nonspecific antiangiogenic effects in models of choroidal neovascularization (4). The consequences of immune activation by siRNA in tumor models was recently illustrated by the potent antitumor effects elicited by both active and nontargeting immune stimulatory siRNA constructs through the activation of immune effector functions (15). The 2'OMe siRNAs developed in our studies induced no measurable cytokine response in mice. There was also no induction of the IFN inducible gene IFIT1 either in the liver, representing the primary target organ for these delivery vehicles, or within secondary lymphoid tissues. IFIT1 expression is responsive to local IFN signaling within tissues and is also induced directly via dsRNA receptors, including TLR3, through an IFN-independent pathway (54-56). Its measure can therefore be considered more broadly indicative of siRNA-mediated immune activation compared with the induction of particular systemic cytokines. Taken together, our results indicate that the appropriate design of $2^{\prime} \mathrm{OMe}$ siRNA can circumvent not only the activation of endosomal TLR7/8 (2, 3, 18, $53)$ but also that of TLR3 (56). We believe that this likely reflects the fact that encapsulation of siRNA within delivery vehicles such as SNALP effectively shields the RNA from exposure to TLR3 on the cell surface. It is important that researchers confirm the full abrogation of an immune response to their selected siRNA in the context of their preferred delivery vehicle and animal model.

A number of strategies for chemically modifying siRNA have been proposed, primarily with the intent to produce nuclease-resistant duplexes (16). From our findings, it is predicted that strategies incorporating 2'OMe-G, 2'OMe-U, or 2'OMe-adenosine (2'OMe-A) residues into both strands of the duplex will generate nonimmunostimulatory siRNA. One such method for siRNA design employs alternating 2 'OMe nucleotides throughout both strands of the duplex (57). Santel and colleagues (58) have tested these 2'OMe siRNAs against the angiogenic target CD31 in tumor models using a lipoplex formulation that transfects vascular endothelium. Antitumor effects in these studies were correlated with specific reductions in CD31 expression and tumor vasculature in the apparent absence of overt immune stimulation. While the authors did not confirm the induction of RNAi in their models and only looked at systemic IFN- $\alpha 24$ hours after siRNA administration, the report represents one of the very few published RNAi studies in oncology to use chemically modified siRNA constructs predicted to have minimal immunostimulatory capacity. It should be noted that this siRNA design is based on blunt-ended 19-mer duplexes that, as naked molecules, are predicted not to activate TLR3 (4). This assumption needs to be formally tested for these lipoplexed siRNAs to ensure that complexing of short siRNA does not enable their engagement of cell-surface TLR3 or other RNA receptors.

Target silencing by siRNA may offer several advantages over functional inhibition by small-molecule drugs. By its nature, RNAi is highly specific and allows for the selective inhibition of closely related proteins compared with the relative promiscuity of kinase inhibitors. Current PLK1 inhibitors, for example, also inhibit PLK2 and PLK3 kinase activity $(23,59)$, raising some concern that concomitant inhibition of these family members may have opposing effects in controlling cell division (60). The biological response to protein depletion by RNAi can also differ from its functional inhibition by small molecules, for example, the loss of both kinase and polo-box functionality upon PLK1 gene silencing (61). The duration of drug effect that can be achieved with siRNA is another attractive advantage. Once RNAi is established within mammalian cells, gene silencing can persist for many days due to the relative stability of activated RISC in the presence of its complementary mRNA $(26,62)$. Therefore, the maintenance of drug activity for an siRNA therapeutic is uncoupled from the requirement to maintain an effective drug concentration 
A

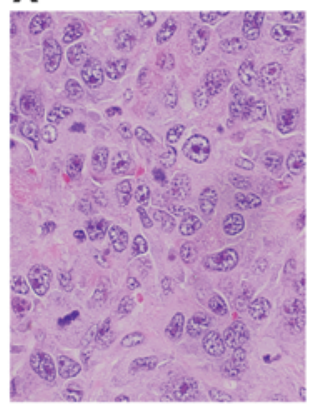

C

B
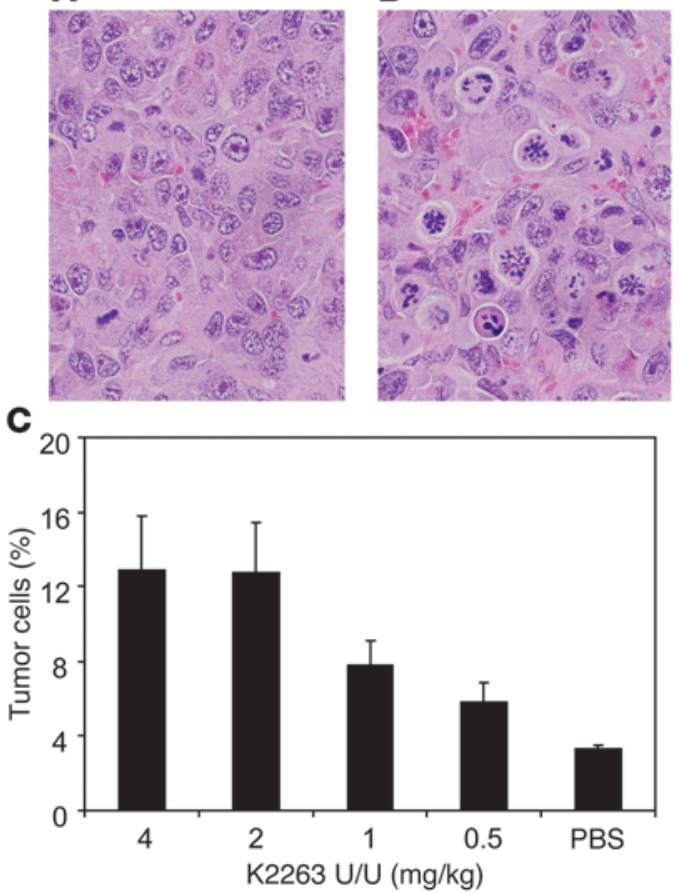

in the blood. We have found that active RNAi in our tumor models persisted for up to 10 days, based on detection of the specific mRNA cleavage product by RACE-PCR. Interestingly, this duration of effect was substantially shorter than that observed in comparable studies targeting ApoB expression in the healthy mouse liver in which silencing at the mRNA level slowly resolved between 14 and 28 days after siRNA administration (ref. 26 and I. MacLachlan, unpublished observations). We believe that the attenuation of RNAi in the tumor most likely results from the effective killing of affected tumor cells and from the dilution of activated RISC through the proliferation of cells receiving sublethal doses of PLK1 siRNA (62).

In conclusion, in this report we have demonstrated that systemic administration of SNALP-formulated siRNA can trigger RNAi-mediated cleavage of mRNA within solid tumors, silencing target expression at a magnitude sufficient to induce the mitotic disruption and apoptosis of tumor cells. We are able to reach this conclusion with the utmost confidence based on the fact that we have followed a clear and rigorous path that allows us to separate siRNA-mediated effects on gene expression from other off-target effects - hence, the importance of this report. Studies are now ongoing to evaluate the utility of using SNALP-formulated siRNA in combination with small-molecule drugs in hopes that this combination may further enhance the efficacy of siRNA molecules in treating human malignancies.

\section{Figure 8}

PLK1424-2A induces distinct phenotypic changes typical of PLK1 inhibition in hepatic tumor cells. H\&E tumor histology 24 hours after single i.v. administration of $2 \mathrm{mg} / \mathrm{kg}$ SNALP-formulated (A and C) LUC-U/U or (B and D) PLK1424-2/A siRNA. Images are representative of tumors from at least 7 individual mice. Original magnification, $\times 200$ (A and B); $\times 400$ (C and D). Mean SNALP particle sizes were 72 (0.04 polydispersity) and 72 (0.02 polydispersity) $\mathrm{nm}$ for PLK1424-2/A and LUC-U/U, respectively.

\section{Figure 7}

KSP2263-U/U induces distinct phenotypic changes typical of KSP inhibition in hepatic tumor cells. Hepatic Neuro2a tumor histology 24 hours after a single i.v. administration of (A) LUC-U/U or (B) KSP2263-U/U siRNA formulated in SNALP (2 mg/kg siRNA). Images representative of tumors from at least 6 individual mice. H\&E staining reveals tumor cells with aberrant nuclear figures typical of monoastral spindles or apoptotic phenotypes in KSP2263-U/U-treated mice. Original magnification, $\times 200$. (C) Quantitative histology of H\&E-stained tumor tissues from mice treated with SNALP-formulated KSP2263-U/U at 4, 2, 1, or $0.5 \mathrm{mg} / \mathrm{kg}$ siRNA. Tumor cells with condensed chromatin structures were scored as positive, and the number of such tumor cells was calculated as a percentage of total tumor cells taken from 10 fields of view. Positive cells included aberrant and typical mitotic and apoptotic figures. Values are mean + SD of 3 mice. Mean SNALP particle sizes were 88 ( 0.07 polydispersity) and 82 ( 0.08 polydispersity) $\mathrm{nm}$ for KSP2263-U/U and LUC-U/U, respectively.

\section{Methods}

siRNA. siRNA sequences targeting hPLK1 (GenBank accession number NM_005030) were selected using a standard siRNA design algorithm $(40,63)$. Target sequences of PLK1 siRNAs are listed in Supplemental Table 1. All siRNAs were synthesized as oligonucleotides by Integrated DNA Technologies and received as desalted, deprotected RNA. Integrity of annealed duplexes was confirmed by $20 \%$ PAGE. siRNAs were formulated into SNALP comprising synthetic cholesterol (Sigma-Aldrich), 1,2-distearoyl-sn-glycero-3-phosphocholine (DSPC; Avanti Polar Lipids Inc.), PEG-cDMA, and 1,2-dilinoleyloxy-3-( $N, N$-dimethyl)aminopropane (DLinDMA) as previously described (26). Formulations used for in vivo studies comprised a final lipid/siRNA mass ratio of 9:1. In the experiments indicated, PEG-cDMA was substituted at equimolar concentra-

A

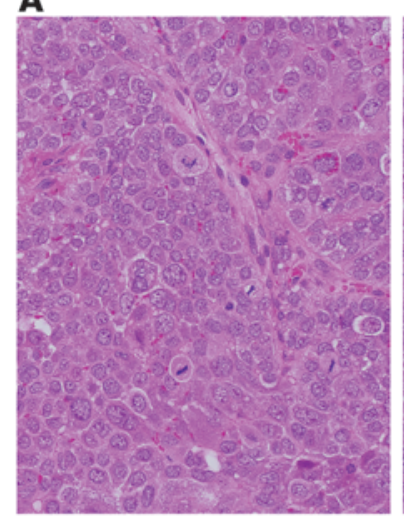

C

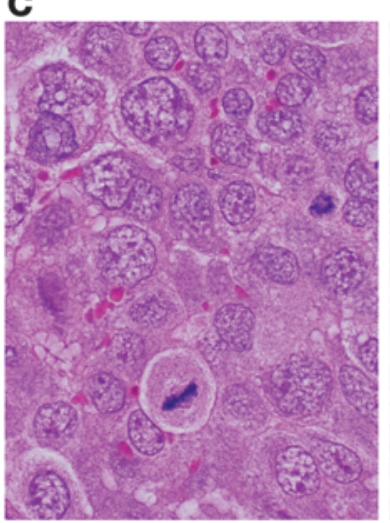

B

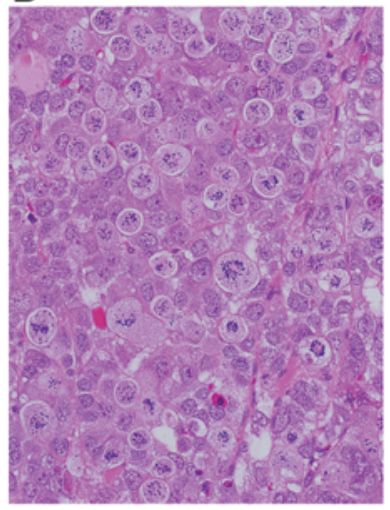

D

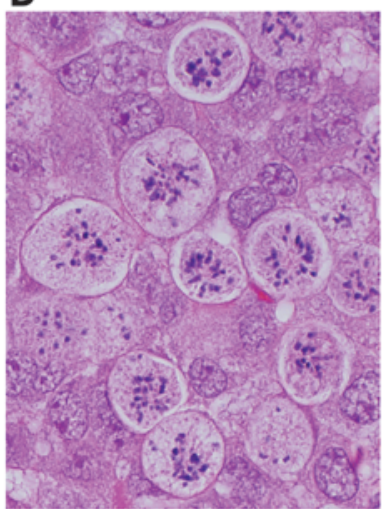


A

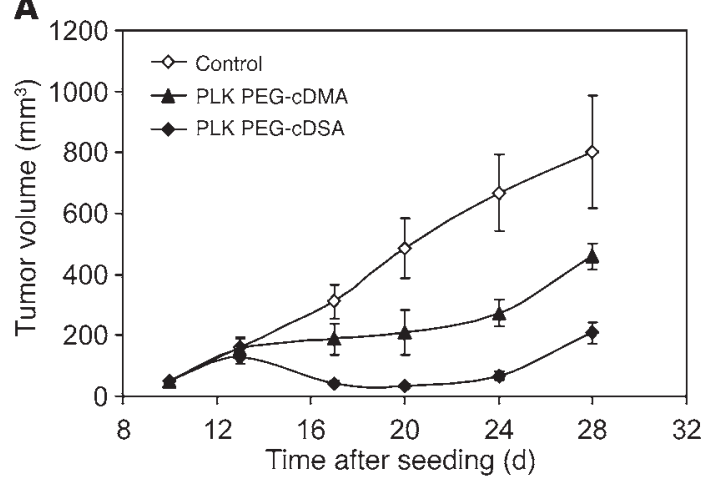

C

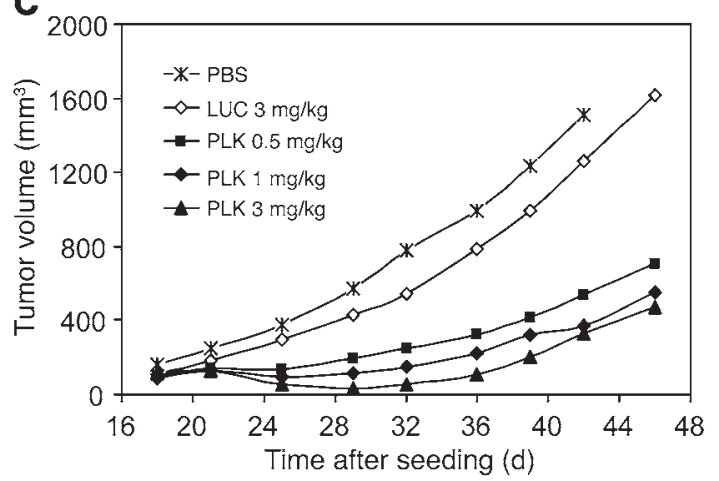

B

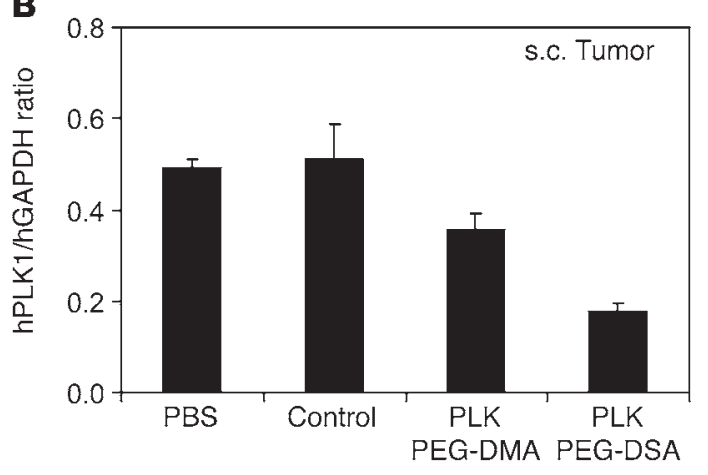

Figure 9

Therapeutic activity of PLK1 SNALP containing either C14 or C18 PEG-lipids in s.c. tumors. (A) Inhibition of s.c. tumor growth by alternate PLK1424-2/A SNALP formulations. Mice were administered PLK1424-2/A SNALP comprising either PEG-cDMA or PEG-cDSA (6 $\times 2$ mg/kg i.v.) between day 10 and day 21 after Hep3B tumor seeding. Values show mean tumor volumes $\left(\mathrm{mm}^{3}\right) \pm \mathrm{SD}(n=5)$. Control was LUC-U/U siRNA SNALP (PEG-cDMA). (B) Corresponding hPLK1/hGAPDH mRNA ratio in s.c. Hep3B tumors following single administration (2 mg/kg) of PLK1424-2/A or LUC-U/U siRNA; mean + SD $(n=4)$. (C) Dose response of PLK1424-2/A PEG-cDSA SNALP in Hep3B tumors. Mice bearing established $\left(\sim 100 \mathrm{~mm}^{3}\right)$ tumors were administered PLK1424-2/A PEG-cDSA SNALP $(6 \times 3,6 \times 1$, or $6 \times 0.5 \mathrm{mg} / \mathrm{kg})$, LUC PEG-cDSA SNALP $(6 \times 3 \mathrm{mg} / \mathrm{kg})$, or PBS vehicle every $2-3$ days between days 18 and 29 after seeding. Values represent mean tumor volumes $\left(\mathrm{mm}^{3}\right)(n=5)$. Mean SNALP particle sizes were 81 ( 0.10 polydispersity), 71 ( 0.03 polydispersity), 82 ( 0.12 polydispersity), and 74 ( 0.05 polydispersity) nm for PLK1424-2/A PEG-cDMA, PEG-cDSA, LUC-U/U PEG-cDMA, and PEG-cDSA, respectively.

tions with the C18 analogue PEG-cDSA (50). All SNALP were dialyzed in PBS prior to use and were stable as a wet preparation stored at $4{ }^{\circ} \mathrm{C}$ for greater than 6 months.

Cell cultures. The cell lines Hep3B, HepG2, HT29, LS174T, and Neuro2a were obtained from ATCC and cultured in the recommended basal medium with $10 \%$ heat-inactivated FBS and $1 \%$ penicillin-streptomycin. For in vivo tumor studies, Hep3B or Neuro2a cells were cultured in T175 flasks, harvested, and washed once in PBS prior to implantation. For in vitro siRNA activity assays, cell lines were cultured in 96-well plates in the presence of SNALP-formulated siRNAs. Cell viability was assessed after 72 hours using the resazurin dye CellTiter-Blue (Promega). Corresponding PLK1 or KSP mRNA-silencing activity was assessed in replicate plates at 24 hours by bDNA assay (Panomics). The level of caspase- 3 and caspase- 7 enzyme activity in siRNA-treated cells was assessed using the fluorescent caspase-3/7 substrate (Z-DEVD)2-Rhodamine 110 reagent Apo-ONE (Promega).

In vitro immune stimulation assays. Mouse Flt $3 \mathrm{~L}$ dendritic cell cultures were generated as described previously (64). In brief, bone marrow from $\mathrm{BALB} / \mathrm{c}$ mice was harvested in complete medium (RPMI 1640, 10\% FBS, $1 \%$ penicillin/streptomycin, $2 \mathrm{mM}$ L-glutamine, $1 \mathrm{mM}$ sodium pyruvate, $25 \mathrm{mM}$ HEPES, and $50 \mu \mathrm{M} 2$-mercaptoethanol), passed through a 70 -micron strainer, and resuspended at $2 \times 10^{6} \mathrm{cells} / \mathrm{ml}$ in complete medium supplemented with $100 \mathrm{ng} / \mathrm{ml}$ murine Flt3L (PeproTech). Cells were seeded in 6-well plates, and $1 \mathrm{ml}$ fresh Flt3L medium was added every 3 days. On day 9 of culture, nonadherent cells were plated into 96-well plates at a concentration of $2 \times 10^{5}$ cells/well. Formulated siRNAs were diluted in PBS and added to the cells for 24 hours before supernatants were assayed for cytokines by ELISA.

In vivo immune stimulation assays. All animal studies were performed at Protiva Biotherapeutics in accordance with Canadian Council on Animal Care guidelines and following protocols approval by the Institutional Animal Care and Use Committee of Protiva Biotherapeutics. Six- to eightweek-old $\mathrm{BALB} / \mathrm{c}$ mice were obtained from Harlan and subjected to a 2-week acclimation period prior to use. Mice were administered SNALPformulated siRNAs $(2 \mathrm{mg} / \mathrm{kg})$ in PBS via standard i.v. injection in the lateral tail vein. Blood was collected by cardiac puncture and processed as plasma for cytokine analysis. Liver and spleen were collected into RNAlater (Sigma-Aldrich) for IFIT1 mRNA analysis.

Intrahepatic tumor models. Liver tumors were established in mice by direct intrahepatic injection of Hep3B or Neuro2a tumor cells (45). Female SCID/beige mice (Charles River) and male A/J mice (Jackson Laboratory) were used as hosts for the Hep3B and Neuro2a tumors, respectively. Animals received Anafen by s.c. injection immediately prior to surgery. Individual mice were anesthetized by isoflurane gas inhalation and eye lube applied to prevent excessive eye drying. While mice were maintained under 
gas anesthesia, a single 1.5 -cm incision across the midline was made below the sternum, and the left lateral hepatic lobe was exteriorized. $1 \times 10^{6}$ Hep3B cells or $1 \times 10^{5}$ Neuro2a cells suspended in $25 \mu \mathrm{l}$ PBS were injected slowly into the lobe at a shallow angle using a Hamilton syringe and a 30 -gauge needle. A swab was then applied to the puncture wound to stop any bleeding prior to suturing. Mice were allowed to recover from anesthesia in a sterile cage and monitored closely for 2-4 hours before being returned to conventional housing.

Eight to eleven days after tumor implantation, mice were randomized into treatment groups. siRNA SNALP formulations or PBS vehicle control was administered by standard i.v. injection via the lateral tail vein, calculated on a $\mathrm{mg}$ siRNAs/kg basis according to individual animal weights $(10 \mathrm{ml} / \mathrm{kg}$ injection volume). Body weights were then monitored throughout the duration of the study as an indicator of developing tumor burden and treatment tolerability. For efficacy studies, defined humane end points were determined as a surrogate for survival. Assessments were made by qualified veterinary technicians based on a combination of clinical signs, weight loss, and abdominal distension to define the day of euthanization due to tumor burden.

s.c. tumor models. Hep3B tumors were established in female SCID/beige mice by s.c. injection of $3 \times 10^{6}$ cells in $50 \mu \mathrm{l}$ PBS into the left-hind flank. Mice were randomized into treatment groups 10-17 days after seeding as tumors became palpable. siRNA SNALP formulations were administered as described above. Tumors were measured in 2 dimensions (width $x$ length) to assess tumor growth using digital calipers. Tumor volume was calculated using the equation $a \times b \times b / 2$, where $a=$ largest diameter and $b=$ smallest diameter, and expressed as group mean \pm SD.

Measurement of hPLK1 and GAPDH mRNA in tumor tissues. Tumors were harvested directly into RNAlater and stored at $4^{\circ} \mathrm{C}$ until processing. $100 \mathrm{mg}$ tumor tissue was homogenized in tissue and lysis solution (EPICENTRE Biotechnologies) containing $50 \mathrm{mg} / \mathrm{ml}$ proteinase K (EPICENTRE Biotechnologies) in a FastPrep tissue homogenizer followed by incubation in a $65^{\circ} \mathrm{C}$ water bath for 15 minutes and centrifugation to clarify lysates. mRNA analysis shown in Figure 5B was performed on purified RNA isolated according to the 5' RACE-PCR protocol. hPLK1 and GAPDH mRNA were measured in tumor lystes by the QuantiGene bDNA assay (Panomics) per the manufacturer's instructions (QuantiGene 1.0 manual). Humanspecific PLK1 (GenBank accession number NM_005030) and GAPDH (GenBank accession number NM_002046) probe sets were designed by Panomics and demonstrated to have minimal cross-reactivity to the mouse counterpart mRNA. Data were expressed as mean PLK1/GAPDH ratio \pm SD of individual animals. Tumor burden was assessed by homogenizing the complete liver from tumor-bearing mice and measuring the total hGAPDH signal (relative light units [RLU]) within the liver. Values were expressed as hGAPDH RLU/mg total liver.

Measurement of IFIT1 mRNA in mouse tissues. Murine liver and spleen were processed for bDNA assay to determine IFIT1 mRNA as described above. The IFIT1 probe set was specific to mouse IFIT1 mRNA (positions 4-499, GenBank accession number NM_008331), and the GAPDH probe set was specific to mouse GAPDH mRNA (positions 9-319, GenBank accession number NM_008084). Data are shown as the ratio of IFIT1 RLU to GAPDH RLU.

5' RLM RACE. Total RNA was isolated from in vitro-cultured cells by direct lysis in TRIzOL (Invitrogen). For in vivo tumor samples, tissues were harvested into RNAlater (Sigma-Aldrich) and stored at $4{ }^{\circ} \mathrm{C}$ for at least 24 hours prior to processing. $30 \mathrm{mg}$ tumor tissue was homogenized in $1 \mathrm{ml}$ TRIzoL, then processed to isolate total RNA. RNA quality was confirmed by gel electrophoresis ( $1 \%$ agarose in Tris-borate buffer). $5^{\prime}$ RNA ligase-mediated-RACE (5' RLM RACE) was performed according to the Invitrogen GeneRacer manual with modifications. Primers were designed using Primer3 software, version 0.3 .0 (http://frodo.wi.mit.edu/). $10 \mu \mathrm{g}$ total RNA was mixed with 1.3 ng GeneRacer RNA adaptor (5' CGACUGGAGCACGAGGACACUGACAUGGACUGAAGGAGUAGAAA $3^{\prime}$ ), heated to $65^{\circ} \mathrm{C}$ for 5 minutes, and snap-cooled on ice prior to ligation. RNA ligation was performed at $37^{\circ} \mathrm{C}$ for 1 hour in $1 \times$ ligase buffer, $30 \mathrm{U}$ RNaseOut (Invitrogen), and $30 \mathrm{U}$ RNA ligase (Ambion Inc.). Samples were then purified by diafiltration using Microcon 100 filters per the manufacturer's instructions for nucleic acids (Millipore). $10 \mu \mathrm{l}$ of the RNA ligation product was reverse transcribed using SuperScript III (Invitrogen) and a PLK1-specific primer (5'-GGACAAGGCTGTAGAACCCACAC-3') designed to hybridize to a target site $3^{\prime}$ to the predicted PLK1424 siRNA-mediated mRNA cut site. Reverse transcription was carried out at $55^{\circ} \mathrm{C}$ for 50 minutes followed by inactivation at $70^{\circ} \mathrm{C}$ for 15 minutes and snap-cooling on ice. $5^{\prime}$ RLM RACE-PCR was performed using forward (GR5) and reverse (PLK1424rev) primers in the GeneRacer adaptor and the 3' end of PLK1 mRNA, respectively, to span the predicted PLK1424 cut site. PCR primer sequences were GR5 5'-CGACTGGAGCACGAGGACACTGA-3' and PLK1424rev 5'-CCAGATGCAGGTGGGAGTGAGGA-3'. PCR was performed using a Bio-Rad iCycler using touchdown PCR conditions of $94^{\circ} \mathrm{C}$ for 2 minutes (1 cycle), $94^{\circ} \mathrm{C}$ for 30 seconds and $72^{\circ} \mathrm{C}$ for 1 minute $(5$ cycles $), 94^{\circ} \mathrm{C}$ for 30 seconds and $70^{\circ} \mathrm{C}$ for 1 minute $\left(5\right.$ cycles), $94^{\circ} \mathrm{C}$ for 30 seconds, $65^{\circ} \mathrm{C}$ for 30 seconds and $68^{\circ} \mathrm{C}$ for 1 minute $\left(25\right.$ cycles), and $68^{\circ} \mathrm{C}$ for 10 minutes $(1 \mathrm{cycle})$. PCR products were run on a $2 \%$ TBE Agarose 1000 (Invitrogen) gel and stained with $1 \mu \mathrm{g} / \mathrm{ml}$ ethidium bromide. The identity of PCR products was confirmed by direct sequencing of the gel-purified products using sequencing primers within the GeneRacer RNA adaptor (5'-ACTGGAGCACGAGGACAC-3') and $3^{\prime}$ PLK1 mRNA (5'-GAGACGGGCAGGGATATAG-3'). Similar assay conditions and primer design were employed to amplify the cleaved KSP mRNA product by KSP2263 siRNA using the following unique primers: KSP-specific cDNA primer 5'-GCTGCTCTCGTGGTTCAGTTCTC-3', RACE primer KSPrev 5'-GCCCAACTACTGCTTAACTGGCAAA-3', and KSP sequencing primer 5'-TGGGTTTCCTTTATTGTCTT-3'.

Histology. Tumors were harvested from mice 24 hours after siRNA administration and fixed directly in $10 \%$ buffered formalin. Tissues were then processed as paraffin-embedded tissue sections and stained with $\mathrm{H} \& \mathrm{E}$ using conventional histological techniques. Quantitative analysis of stained sections was performed by counting the number of mitotic/apoptotic cells displaying condensed chromatin structures as a percentage of total tumor cells. Values for each tumor were derived from means of 10 fields of view at $\times 400$ magnification.

Cytokine ELISA. All cytokines were quantified using sandwich ELISA kits. These were mouse IFN- $\alpha$ (PBL) and human and mouse IL-6 (BD Biosciences).

Statistics. Comparisons of survival times were performed on KaplanMeier plots by the log-rank (Cox-Mantel) test. Differences were deemed significant at $P<0.05$.

\section{Acknowledgments}

The authors would like to acknowledge Ed Yaworski, Lloyd Jeffs, Lina Huang, Felix Yuen, and James Heyes for siRNA formulation and lipid synthesis. I. Tavakoli, J. Levi, Lina Hu, and A. Fronda were supported by undergraduate research grants from the Natural Sciences and Engineering Research Council of Canada.

Received for publication September 22, 2008, and accepted in revised form December 17, 2008.

Address correspondence to: Ian MacLachlan, Tekmira Pharmaceuticals Corporation, 100-8900 Glenlyon Parkway, Burnaby, British ColumbiaV5J5J8, Canada. Phone:(604) 419-3205; Fax:(604) 419-3201; E-mail: imaclachlan@tekmirapharm.com. 
1. Elbashir, S.M., et al. 2001. Duplexes of 21-nucleotide RNAs mediate RNA interference in cultured mammalian cells. Nature. 411:494-498.

2. Judge, A.D., et al. 2005. Sequence-dependent stimulation of the mammalian innate immune response by synthetic siRNA. Nat. Biotechnol. 23:457-462.

3. Hornung, V., et al. 2005. Sequence-specific potent induction of IFN-alpha by short interfering RNA in plasmacytoid dendritic cells through TLR7. Nat Med. 11:263-270.

4. Kleinman, M.E., et al. 2008. Sequence and targetindependent angiogenesis suppression by siRNA via TLR3. Nature. 452:591-597.

5. Hornung, V., et al. 2006. 5'-Triphosphate RNA is the ligand for RIG-I. Science. 314:994-997.

6. Marques, J.T., et al. 2006. A structural basis for discriminating between self and nonself doublestranded RNAs in mammalian cells. Nat. Biotechnol. 24:559-565.

7. Krieg, A.M. 2006. Therapeutic potential of Tolllike receptor 9 activation. Nat. Rev. Drug Discov. 5:471-484

8. von Marschall, Z., et al. 2003. Effects of interferon alpha on vascular endothelial growth factor gene transcription and tumor angiogenesis. J. Natl. Cancer Inst. 95:437-448.

9. Lanuti, M., et al. 2000. Cationic lipid:bacterial DNA complexes elicit adaptive cellular immunity in murine intraperitoneal tumor models. Cancer Res. 60:2955-2963.

10. Buhtoiarov, I.N., Lum, H.D., Berke, G., Sondel, P.M., and Rakhmilevich, A.L. 2006. Synergistic activation of macrophages via CD40 and TLR9 results in $\mathrm{T}$ cell independent antitumor effects. J. Immunol. 176:309-318

11. Judge, A., and MacLachlan, I. 2008. Overcoming the innate immune response to small interfering RNA. Hum. Gene Ther. 19:111-124.

12. Michie, H.R., et al. 1988. Detection of circulating tumor necrosis factor after endotoxin administration. N. Engl. J. Med. 318:1481-1486.

13. Stevenson, H.C., et al. 1985. A phase I evaluation of poly(I,C)-LC in cancer patients. J. Biol. Response Mod. 4:650-655.

14. Schlee, M., Barchet, W., Hornung, V., and Hartmann, G. 2007. Beyond double-stranded RNA-type I IFN induction by 3pRNA and other viral nucleic acids. Curr. Top. Microbiol. Immunol. 316:207-230.

15. Poeck, H., et al. 2008. 5'-triphosphate-siRNA: turning gene silencing and Rig-I activation against melanoma. Nat. Med. 14:1256-1263.

16. Manoharan, M. 2004. RNA interference and chemically modified small interfering RNAs. Curr. Opin. Chem. Biol. 8:570-579.

17. Morrissey, D.V., et al. 2005. Potent and persistent in vivo anti-HBV activity of chemically modified siRNAs. Nat. Biotechnol. 23:1002-1007.

18. Judge, A.D., Bola, G., Lee, A.C., and MacLachlan, I. 2006. Design of non-inflammatory synthetic siRNA mediating potent gene silencing in vivo. Mol. Ther. 13:494-505.

19. Knight, S.D., and Parrish, C.A. 2008. Recent progress in the identification and clinical evaluation of inhibitors of the mitotic kinesin KSP. Curr. Top. Med. Chem. 8:888-904.

20. Strebhardt, K., and Ullrich, A. 2006. Targeting pololike kinase 1 for cancer therapy. Nat. Rev. Cancer. 6:321-330.

21. Tao, W., et al. 2005. Induction of apoptosis by an inhibitor of the mitotic kinesin KSP requires both activation of the spindle assembly checkpoint and mitotic slippage. Cancer Cell. 8:49-59.

22. Barr, F.A., Sillje, H.H., and Nigg, E.A. 2004. Pololike kinases and the orchestration of cell division. Nat. Rev. Mol. Cell Biol. 5:429-440.

23. Steegmaier, M., et al. 2007. BI 2536, a potent and selective inhibitor of polo-like kinase 1 , inhibits tumor growth in vivo. Curr. Biol. 17:316-322.

24. Liu, X., and Erikson, R.L. 2003. Polo-like kinase (Plk)1 depletion induces apoptosis in cancer cells. Proc. Natl. Acad. Sci. U. S. A. 100:5789-5794.

25. Spankuch, B., Kurunci-Csacsko, E., Kaufmann, M., and Strebhardt, K. 2007. Rational combinations of siRNAs targeting Plk1 with breast cancer drugs. Oncogene. 26:5793-5807.

26. Zimmermann, T.S., et al. 2006. RNAi-mediated gene silencing in non-human primates. Nature. 441:111-114.

27. de Fougerolles, A., Vornlocher, H.P., Maraganore, J., and Lieberman, J. 2007. Interfering with disease: a progress report on siRNA-based therapeutics. Nat. Rev. Drug Discov. 6:443-453.

28. Behlke, M.A. 2006. Progress towards in vivo use of siRNAs. Mol. Ther. 13:644-670.

29. Urban-Klein, B., Werth, S., Abuharbeid, S., Czubayko, F., and Aigner, A. 2005. RNAi-mediated gene-targeting through systemic application of polyethylenimine (PEI)-complexed siRNA in vivo. Gene Ther. 12:461-466.

30. Schiffelers, R.M., et al. 2004. Cancer siRNA therapy by tumor selective delivery with ligand-targeted sterically stabilized nanoparticle. Nucleic Acids Res. 32:e149.

31. Heidel, J.D., et al. 2007. Administration in nonhuman primates of escalating intravenous doses of targeted nanoparticles containing ribonucleotide reductase subunit M2 siRNA. Proc. Natl. Acad. Sci. U. S. A. 104:5715-5721.

32. Santel, A., et al. 2006. A novel siRNA-lipoplex technology for RNA interference in the mouse vascular endothelium. Gene Ther. 13:1222-1234.

33. Mayer, L., Bally, M., Cullis, P., Wilson, S., and Emerman, J. 1990. Comparison of free and liposome encapsulated doxorubicin tumor drug uptake and anti-tumor efficacy in the SC115 murine mammary tumor. Cancer Letters. 53:183-190.

34. Seymour, L.W. 1992. Passive tumor targeting of soluble macromolecules and drug conjugates. Crit. Rev. Ther. Drug Carrier Syst. 9:135-187.

35. Chono, S., Li, S.D., Conwell, C.C., and Huang, L. 2008. An efficient and low immunostimulatory nanoparticle formulation for systemic siRNA delivery to the tumor. J. Control Release. 131:64-69.

36. Peer, D., Park, E.J., Morishita, Y., Carman, C.V., and Shimaoka, M. 2008. Systemic leukocyte-directed siRNA delivery revealing cyclin D1 as an antiinflammatory target. Science. 319:627-630.

37. Robbins, M., et al. 2008. Misinterpreting the therapeutic effects of siRNA caused by immune stimulation. Hum Gene Ther. Online publication ahead of print. doi:10.1089/hgt.2008.131.

38. Weil, D., et al. 2002. Targeting the kinesin Eg5 to monitor siRNA transfection in mammalian cells. Biotechniques. 33:1244-1248.

39. Soutschek, J., et al. 2004. Therapeutic silencing of an endogenous gene by systemic administration of modified siRNAs. Nature. 432:173-178.

40. Elbashir, S.M., Lendeckel, W., and Tuschl, T. 2001. RNA interference is mediated by 21 - and 22 -nucleotide RNAs. Genes Dev. 15:188-200.

41. Matranga, C., Tomari, Y., Shin, C., Bartel, D.P., and Zamore, P.D. 2005. Passenger-strand cleavage facilitates assembly of siRNA into Ago2-containing RNAi enzyme complexes. Cell. 123:607-620.

42. Leuschner, P.J., Ameres, S.L., Kueng, S., and Martinez, J. 2006. Cleavage of the siRNA passenger strand during RISC assembly in human cells. EMBO Rep. 7:314-320.

43. Prakash, T., et al. 2005. Positional effect of chemical modifications on short interference RNA activity in mammalian cells. J. Med. Chem. 48:4247-4253.

44. Chiu, Y., and Rana, T. 2003. siRNA function in
RNAi: A chemical modification analysis. RNA. 9:1034-1048.

45. Yao, X., et al. 2003. A novel orthotopic tumor model to study growth factors and oncogenes in hepatocarcinogenesis. Clin. Cancer Res. 9:2719-2726.

46. Sakowitz, R., et al. 2004. Antitumor activity of kinesin inhibitor. Cancer Res. 64:3276-3280.

47. Lenart, P., et al. 2007. The small-molecule inhibitor BI 2536 reveals novel insights into mitotic roles of polo-like kinase 1. Curr. Biol. 17:304-315.

48. Spankuch-Schmitt, B., Bereiter-Hahn, J., Kaufmann, M., and Strebhardt, K. 2002. Effect of RNA silencing of polo-like kinase-1 (PLK1) on apoptosis and spindle formation in human cancer cells. J. Natl. Cancer Inst. 94:1863-1877.

49. Ambegia, E., et al. 2005. Stabilized plasmid-lipid particles containing PEG-diacylglycerols exhibit extended circulation lifetimes and tumor selective gene expression. Biochim. Biophys. Acta. 1669:155-163.

50. Heyes, J., Hall, K., Tailor, V., Lenz, R., and MacLachlan, I. 2006. Synthesis and characterisation of novel poly(ethylene glycol)-lipid conjugates suitable for use in drug delivery. J. Control. Release. 112:280-290.

51. Krieg, A.M. 2002. CpG motifs in bacterial DNA and their immune effects. Annu. Rev. Immunol. 20:709-760.

52. Castro, J.E., et al. 2006. Thymidine-phosphorothioate oligonucleotides induce activation and apoptosis of CLL cells independently of CpG motifs or BCL-2 gene interference. Leukemia. 20:680-688.

53. Diebold, S.S., et al. 2006. Nucleic acid agonists for Toll-like receptor 7 are defined by the presence of uridine ribonucleotides. Eur. J. Immunol. 36:3256-3267.

54. Der, S.D., Zhou, A., Williams, B.R., and Silverman, R.H. 1998. Identification of genes differentially regulated by interferon alpha, beta, or gamma using oligonucleotide arrays. Proc. Natl. Acad. Sci. U. S. A. 95:15623-15628.

55. Geiss, G., et al. 2001. A comprehensive view of regulation of gene expression by double-stranded RNA-mediated cell signaling. J. Biol. Chem. 276:30178-30182.

56. Sarkar, S.N., Smith, H.L., Rowe, T.M., and Sen, G.C. 2003. Double-stranded RNA signaling by Toll-like receptor 3 requires specific tyrosine residues in its cytoplasmic domain. J. Biol. Chem. 278:4393-4396.

57. Czauderna, F., et al. 2003. Structural variations and stabilising modifications of synthetic siRNAs in mammalian cells. Nucl. Acids Res. 31:2705-2716.

58. Santel, A., et al. 2006. RNA interference in the mouse vascular endothelium by systemic administration of siRNA-lipoplexes for cancer therapy. Gene Ther. 13:1360-1370.

59. Johnson, E.F., Stewart, K.D., Woods, K.W., Giranda, V.L., and Luo, Y. 2007. Pharmacological and functional comparison of the polo-like kinase family: insight into inhibitor and substrate specificity. Biochemistry. 46:9551-9563.

60. Malumbres, M., and Barbacid, M. 2007. Cell cycle kinases in cancer. Curr. Opin. Genet. Dev. 17:60-65.

61. McInnes, C., et al. 2006. Inhibitors of Polo-like kinase reveal roles in spindle-pole maintenance. Nat. Chem. Biol. 2:608-617.

62. Bartlett, D.W., and Davis, M.E. 2006. Insights into the kinetics of siRNA-mediated gene silencing from live-cell and live-animal bioluminescent imaging. Nucleic Acids Res. 34:322-333.

63. Schwarz, D.S., et al. 2003. Asymmetry in the assembly of the RNAi enzyme complex. Cell. 115:199-208

64. Gilliet, M., et al. 2002. The development of murine plasmacytoid dendritic cell precursors is differentially regulated by FLT3-ligand and granulocyte/ macrophage colony-stimulating factor. J. Exp. Med. 195:953-958. 